Research Paper

\title{
Downregulation of $\mathrm{m}^{6} \mathrm{~A}$ Reader YTHDC2 Promotes the Proliferation and Migration of Malignant Lung Cells via CYLD/NF-kB Pathway
}

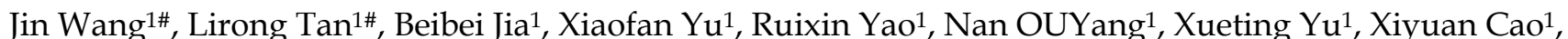
Jian Tong ${ }^{1}$, Tao Chen ${ }^{1}$, Rui Chen ${ }^{2}$ and Jianxiang $\mathrm{Li}^{1 凶}$

1. Department of Toxicology, School of Public Health, Medicine College, Soochow University, Suzhou, Jiangsu, 215123, China.

2. Department of Respiratory Medicine, The Second Affiliated Hospital of Soochow University, Suzhou Jiangsu, 215004, China

\#These authors contributed equally to this work.

$\triangle$ Corresponding authors: Jianxiang Li, E-mail: aljxcr@suda.edu.cn; Rui Chen, E-mail: chenruigood@126.com.

(C) The author(s). This is an open access article distributed under the terms of the Creative Commons Attribution License (https://creativecommons.org/licenses/by/4.0/). See http://ivyspring.com/terms for full terms and conditions.

Received: 2021.02.03; Accepted: 2021.06.08; Published: 2021.06 .22

\begin{abstract}
Lung cancer is one of the most common types of carcinoma worldwide. Cigarette smoking is considered the leading cause of lung cancer. Aberrant expression of several YT521-B homology (YTH) family proteins has been reported to be closely associated with multiple cancer types. The present study aims to evaluate the function and regulatory mechanisms of the N6-methyladenosine $\left(\mathrm{m}^{6} \mathrm{~A}\right)$ reader protein $\mathrm{YTH}$ domain containing 2 (YTHDC2) by in vitro, in vivo and bioinformatics analyses. The results revealed that YTHDC2 was reduced in lung cancer and cigarette smoke-exposed cells. Notably, bioinformatics and tissue arrays analysis demonstrated that decreased YTHDC2 was highly associated with smoking history, pathological stage, invasion depth, lymph node metastasis and poor outcomes. The in vivo and in vitro studies revealed that YTHDC2 overexpression inhibited the proliferation and migration of lung cancer cells as well as tumor growth in nude mice. Furthermore, YTHDC2 decreased expression was modulated by copy number deletion in lung cancer. Importantly, the cylindromatosis (CYLD)/NF-KB pathways were confirmed as the downstream signaling of YTHDC2, and this axis was mediated by $\mathrm{m}^{6} \mathrm{~A}$ modification. The present results indicated that smoking-related downregulation of YTHDC2 was associated with enhanced proliferation and migration in lung cancer cells, and appeared to be regulated by DNA copy number variation. Importantly, YTHDC2 functions as a tumor suppressor through the CYLD/NF-KB signaling pathway, which is mediated by $\mathrm{m}^{6} \mathrm{~A}$ modification.
\end{abstract}

Key words: lung cancer, YTHDC2, m6 RNA methylation, CYLD, NF-kB pathway

\section{Introduction}

Lung cancer is one of the most common types of carcinoma in the world [1]. The two main lung cancer types are small cell lung cancer (SCLC) and non-small cell lung cancer (NSCLC). NSCLC accounts for $85-90 \%$ of all lung cancer cases, and its two most common subtypes are lung squamous cell carcinoma (LUSC) and lung adenocarcinoma (LUAD) [2]. Despite significant advances in early diagnosis and treatment, the 5-year relative overall survival (OS) of lung cancer patient is $<20 \%$ [3]. Smoking is the main cause of lung cancer of all major histological types, and the risk of lung cancer in persistent smokers is 20-50-fold higher than that of non-smokers [4]. A previous study reported that a decreased relative risk and favorable prognosis were found in reformed smokers [5]. Numerous candidate biomarkers contribute to the initialization and progress of lung cancer have been reported in bioinformatics analyses [6-8]. Therefore, the association between smoking and lung cancer, including the significance of differentially expressed genes caused by smoking, and its role in the development of lung cancer, should be further investigated [9]. A previous study suggested that the potency of cigarettes as 
carcinogens may be due to their ability to induce aberrant DNA methylation and altered gene expression, resulting in cells escaping from apoptosis and undergoing subsequent neoplastic transformation [10, 11].

Previous reports suggested that the dysregulation of N6-methyladenosine $\left(\mathrm{m}^{6} \mathrm{~A}\right)$ modifications exerts oncogenic and tumorsuppressive effects in the genesis and development of several cancer types, including lung cancer, liver cancer and acute myeloid leukemia [12-14]. Several proteins in the YT521-B homology (YTH) family were shown to be capable of selectively binding to and processing precursor RNA containing $\mathrm{m}^{6} \mathrm{~A}$ in the nucleus, including YTH N6-Methyladenosine RNA binding protein (YTHDF)1, YTHDF2, YTHDF3, YTH domain containing (YTHDC) 1 and YTHDC2, which are considered to be $\mathrm{m}^{6} \mathrm{~A}$ reader proteins $[15,16]$. These readers can promote efficient translation and degradation of specific $\mathrm{m}^{6} \mathrm{~A}$-containing mRNA, causing rapid changes in gene expression profiles [17, 18]. The increase in $\mathrm{m}^{6} \mathrm{~A}$ methyl-transferase activity was consistently associated with the malignant transformation of cells [19]. Among these $\mathrm{m}^{6} \mathrm{~A}$ readers, YTHDF1 was reported to be associated with hepatocellular carcinoma (HCC) by promoting the Wnt/ $\beta$-catenin signaling pathway [20]. Several reports demonstrated that YTHDF2 was upregulated in HCC [21] and pancreatic cancer [22]. YTHDC2, which is different from the other family members, contains other putative RNA-binding domains in addition to its YTH domain. In addition, YTHDC2 can enhance translation efficiency and decrease the target mRNA abundance by preferentially binding to $\mathrm{m}^{6} \mathrm{~A}$ within the consensus motif [23, 24]. YTHDC2 plays a vital role in spermatogenesis by interacting with meiosis-specific coiled-coil domain-containing protein, thereby affecting the stability of target transcripts in pre-meiosis [25]. However, the precise association between YTHDC2 and cancer remains unclear. It has been reported that upregulated YTHDC2 contributes to the metastasis of colon cancer cells in vivo by promoting the translation of cell migration-related genes [26]. It has been demonstrated that the downregulation of YTHDC2 can inhibit HCC cell proliferation by reducing the translation of mRNAs involved in cell proliferation [27]. However, the role of YTHDC2 in other types of tumors, particularly lung cancer, remains unresolved.

The purpose of this study was to evaluate the expression profile of YTHDC2, a binding-protein of RNA $\mathrm{m}^{6} \mathrm{~A}$ methylation, and to determine its in vitro effects on cigarette smoke (CS)-induced malignant transformed BEAS-2B cells and lung cancer cell behavior, as well as the clinical relevance and regulatory mechanism of YTHDC2.

\section{Materials and methods}

\section{Data analysis from The Cancer Genome Atlas (TCGA) and Gene Expression Omnibus (GEO) datasets}

The $\quad \log _{2}$-transformed RNA-sequencing expression value and copy number alterations of lung cancer in TCGA database, including lung adenocarcinoma (TCGA LUAD) and lung squamous cell carcinoma (TCGA LUSC), were obtained from the University of California Santa Cruz Xena Browser (http://xena.ucsc.edu). Expression of YTHDC2 mRNA was analyzed based on an online tool, Gene Expression Profiling Interactive Analysis (GEPIA; http://gepia.cancer-pku.cn/index.html), which is a newly developed interactive web server for analyzing the RNA-sequencing expression data of 23 types of cancer and normal samples from TCGA and Genotype-Tissue Expression (GTEx) projects according to the standard processing pipeline [28]. Two datasets, GSE32665 [29] and GSE19188 [30], were download from the GEO database (http://www.ncbi.nlm.nih.gov/geo/) for differential expression analysis. Another two datasets, GSE41271 [31] and GSE30219 [32], were downloaded for clinicopathological correlation and survival analyses. The characteristics of the datasets used in the present study are displayed in Table S1. At the protein level, the expression of YTHDC2 in normal and malignant lung tissue was reviewed by data mining in the Human Protein Atlas (HPA; http://www. proteinatlas.org), which included 3 normal lung samples and 10 lung cancer samples. Images of YTHDC2 protein immunohistochemistry (IHC) staining in normal lung tissues and lung cancer tissues were downloaded from this database.

\section{IHC of tissue array}

Human lung cancer tissue arrays (cat. no. LAC-1402 and LAC-1403) were purchased from Wuhan Servicebio Technology Co., Ltd. The tissue arrays consisted of 70 pairs of lung cancer and adjacent normal tissues. IHC assay was used to analyze the expression of YTHDC2 in lung cancer and paired normal tissues with an anti-YTHDC2 antibody (1:500 dilusion; ProteinTech Group, Inc.). ImageJ and ImageJ plug-in IHC Profiler were applied for the quantification of IHC staining [33]. The positive staining ratio was expressed as the sum of the high, low and positive rates.

\section{Survival analysis}

The association between the mRNA expression levels of the YTHDC2 gene and the OS of patients 
with lung cancer was analysed using the online Kaplan-Meier plotter (http:// kmplot.com/analysis/) [34]. In addition to smoking history, other parameters were set to default values. Survival information and YTHDC2 expression data of smokers and non-smokers were obtained according to their smoking history. For the survival analysis of the GSE41271, GSE30219 and TCGA lung cancer datasets, the patients were classified into high $(\geq 75 \%)$ and low $(<75 \%)$ YTHDC2 expression groups according to the $\%$ of YTHDC2 mRNA expression levels. Univariate Cox models were used to calculate the hazard ratios (HRs) and 95\% confidence intervals (CIs). Cox analysis was performed using the 'survival' (https://cran.r-project.org/web/packages/survival/ index.html) package, and the 'survminer' (https:// github.com/kassambara/survminer) package was used to generate Kaplan-Meier survival curves.

\section{Correlation and enrichment analysis}

LinkedOmics (http://linkedomics.org/) is a newly developed web portal based on multi-omics datasets from 32 cancer types in TCGA database [35]. The present study applied this portal to identify the genes listed as positively or negatively correlated with YTHDC2 in the LUAD and LUSC TCGA datasets. These correlated genes were further subjected to enrichment analysis, which was performed using the online enrichment tool DAVID (https:// david.ncifcrf. gov) [36]. TSGene (https://bioinfo.uth.edu/ TSGene/) is a web database for tumour suppressor genes (TSGs) collected from the published literature [37]. Human Cancer Metastasis Database (HCMDB; http://hcmdb.i-sanger.com/index) is an integrated database that has annotated multiple potentially metastasis-related genes (MRGs) [38]. These two databases were used to identify TSGs and MRGs among the YTHDC2-related genes obtained from the LinkedOmics database.

\section{In vitro cell model of CS-induced malignant transformation}

Normal human bronchial epithelial cells (BEAS-2B) were obtained from the American Type Culture Collection and used to establish an in vitro model of CS-induced malignant transformation, as previously described [10]. Briefly, aliquots of exponentially growing $1 \times 10^{5}$ BEAS- $2 \mathrm{~B}$ cells were plated onto a Transwell membrane $(0.4-\mu \mathrm{m}$ pore; Corning, Inc.). An automatic smoking machine was used to produce CS, which was then pumped into an inhalation chamber where the BEAS-2B cells were directly exposed to CS for 10 min every other day at a smoke concentration of 20\% (MED8170A, Tianjin Hope Industry \& Trade Co., Ltd). This procedure was performed to expose the cells to CS for 10, 20 and 30 passages, and such cells were referred as experimental S10, S20 and S30 cells, respectively. The unexposed BEAS-2B cells were used as control cells.

\section{Cell culture and transfection}

Human NSCLC H1299 cells were obtained from the American Type Culture Collection. The cells were cultured in Dulbecco's modified Eagle's medium (DMEM) supplemented with $10 \%$ fetal bovine serum (FBS) in a humidified incubator with $5 \% \mathrm{CO}_{2}$ at $37^{\circ} \mathrm{C}$. The full-length of the YTHDC2 coding sequence was amplified using 2XEasyPfu PCR SuperMix (Beijing Transgen Biotech Co., Ltd.) and cloned into a pCDH vector, and the recombinant plasmid was named pCDH-YTHDC2. The design of shRNAs of $\mathrm{m}^{6} \mathrm{~A}$ methylases METTL3/14 and demethylases FTO/ ALKBH5 relied on the algorithm of an online program BLOCK-iT RNAi Designer (https://rnaidesigner. thermofisher.com/rnaiexpress/). The shRNA oligo pairs were annealed and then inserted into GreenPuro vectors separately. YTHDC2 small interfering RNA (siRNA) was obtained from Guangzhou RiboBio Co., Ltd. The lentiviruses were packaged according to the manufacturer's protocol. Briefly, supernatants containing YTHDC2 lentivirus generated in 293T cells were collected at 48 and $72 \mathrm{~h}$ post-transfection. BEAS-2B, H1299 and S30 cells were transfected with pCDH-YTHDC2 and siRNA using Lipofectamine 6000 (Beyotime Institute of Biotechnology) according to the manufacturer's instructions.

\section{Tandem mass tagging (TMT) proteomics profiling analysis}

Protein samples were extracted from normal BEAS-2B and YTHDC2-knockdown cells. The specific proteomic pipeline is shown in the Supplementary methods. The false discovery rate was adjusted to $<1 \%$ and the minimum score for modified peptides was set to $>40$. A ratio $>1.2$ was considered upregulation, and $<0.83$ was considered downregulation. The differentially expressed proteins were depicted in the STRING database to generate protein-protein interaction network and identify clusters by plug-in Molecular Complex Detection (MCODE) in Cytoscape (https:// cytoscape.org/) [39].

\section{Reverse transcription-quantitative PCR (RT-qPCR)}

Total RNA of samples was isolated using TRIzol ${ }^{\circledR}$ reagent (Invitrogen; Thermo Fisher Scientific, Inc.) according to the manufacturer's protocol. Total RNA $(\sim 1.5 \mu \mathrm{g})$ from each sample was reverse transcribed into cDNA using a Revert Aid First Strand Complementary DNA Synthesis kit (Thermo Fisher 
Scientific, Inc.) according to the manufacturer's instructions. RT-qPCR was performed using an RT-qPCR kit (Novoprotein Scientific, Inc.) on a QuantStudio $^{\mathrm{TM}} 6$ Flex Real-Time PCR system (Applied Biosystems; Thermo Fisher Scientific, Inc.). GAPDH was used as a reference. The primer pairs used for qPCR in the study is listed in Table S2. The relative expression of each target was analyzed using the $2^{-\Delta \Delta \mathrm{Ct}}$ method [40].

\section{Western blot analysis}

Total protein was extracted with RIPA buffer (Beyotime, Institute of Biotechnology) and quantified with BCA assay (Beyotime, Institute of Biotechnology), and $20 \mu \mathrm{g}$ protein was separated on SDS-PAGE and transferred onto a PVDF membrane (MilliporeSigma). Nuclear and cytoplasm proteins were isolated using the Nuclear and Cytoplasmic Protein Extraction kit (CWBio) according to the manufacturer's protocol. After blocking with 5\% bovine serum albumin (BSA), the membrane was incubated at $4{ }^{\circ} \mathrm{C}$ overnight with specific antibodies against YTHDC2 (1:5,000 dilution, Abcam), E-cadherin (CDH1; 1:1,000 dilution; ProteinTech Group, Inc.), N-cadherin (CDH2; 1:1,000 dilution; ProteinTech Group, Inc.), cylindromatosis (CYLD; 1:1,000 dilution; ProteinTech Group, Inc.), NF-kB P65 (1:1,000 dilution; ProteinTech Group, Inc.) and GAPDH (1:1,000 dilution; Cell Signaling Technology, Inc.). HRP-labeled secondary antibodies were used according to the host species of the primary antibodies. The western blot bands were developed using an ECL substrate and visualized using the GeneTools GBOX system (Syngene International). The intensity of each band was quantified using ImageJ software (Version 1.8.0).

\section{Cell proliferation assays}

For cell cycle analysis, the transfected cells were trypsinized and then washed twice with pre-cold PBS. Cells were fixed in $70 \%$ ethanol at $-20{ }^{\circ} \mathrm{C}$ overnight, washed twice with PBS, and treated with $10 \mu \mathrm{g} / \mathrm{ml}$ RNase A (Beyotime Institute of Biotechnology) and 50 $\mu \mathrm{g} / \mathrm{ml}$ propidium iodide (Beyotime Institute of Biotechnology) for $30 \mathrm{~min}$ at $37^{\circ} \mathrm{C}$. Cell cycle analysis was performed using a BD FACSCanto $^{\text {TM }}$ Cell Analyzer (BD Biosciences), and the percentage of cells in the different phases of the cell cycle was calculated by FlowJo v10.3 software (FlowJo LLC).

For the 5-ethynyl-2'-deoxyuridine (EdU) assay, logarithmic growth stage cells $\left(2 \times 10^{5}\right)$ were seeded into a 6-well plate with $10 \mu \mathrm{M}$ EdU reagent for $2 \mathrm{~h}$. After fixing with $4 \%$ paraformaldehyde for $30 \mathrm{~min}$, cells were permeabilized with $0.3 \%$ Triton X-100 in PBS, and incubated with Click-iT reaction solution (cat. no. C0075S; Beyotime Institute of Biotechnology). Images were collected at $24 \mathrm{~h}$ under an inverted fluorescent microscope and quantitatively analyzed with NIH ImageJ software (Version 1.8.0).

\section{Immunofluorescence assay}

Cells were seeded onto glass coverslips placed in 6 -well plates. After fixing with $4 \%$ paraformaldehyde for $30 \mathrm{~min}$, the cells were permeabilized with $0.3 \%$ Triton X-100 in PBS and blocked with 1\% BSA. Next, the cells were incubated with anti-Ki67 polyclonal antibody (1:500 dilution; ProteinTech Group, Inc.) for $1 \mathrm{~h}$ at $37^{\circ} \mathrm{C}$. Subsequently, the cells were incubated with an Alexa Fluor ${ }^{\circledR}$ 448-conjugated secondary antibody (1:1,000 dilution; Cell Signaling Technology, Inc.) for $30 \mathrm{~min}$ and stained with DAPI (Beyotime Institute of Biotechnology) for 5 min protect from light at room temperature. Images were collected under an inverted fluorescent microscope and quantitatively analyzed with NIH ImageJ software (Version 4.0.4).

\section{Cell migration assays}

For scratch wound healing assay, cells $\left(2 \times 10^{5}\right)$ were seeded into 6-well plates, cultured at $37^{\circ} \mathrm{C}$ and allowed to grow to $100 \%$ confluence. Next, a scratch was made in the plate using a P10 pipette tip. The cells were cultured in fresh serum-free DMEM. Images were collected at 0 and $24 \mathrm{~h}$ under an inverted microscope (Olympus Corporation) and quantitatively analyzed with NIH ImageJ software (Version 4.0.4).

For the Transwell migration assay, cells $\left(2 \times 10^{5}\right)$ were seeded in the upper chambers (pore size, $8 \mu \mathrm{m}$ ) of a 6-well plate (Corning, Inc.) with $1 \mathrm{ml}$ serum-free medium. A total of $2 \mathrm{ml}$ complete medium with $10 \%$ FBS was added to the lower chambers, and the plate was incubated for $24 \mathrm{~h}$. Next, the cells in the upper surface of the membrane were removed, and the cells on the lower surface were fixed with $4 \%$ paraformaldehyde and stained with $0.5 \%$ crystal violet solution. Images were obtained and analyzed using NIH ImageJ software (Version 4.0.4).

\section{Xenograft model}

Five-week-old male BALB/c-nude mice were obtained from Beijing Vital River Laboratory Animal Technology Co., Ltd. All animals were housed in the Laboratory Animal Center of Soochow University and cared for in accordance with the NIH Guide for the Care and Use of Laboratory Animals. The experimental protocol was approved by the Laboratory Animal Ethics Committee of the Experimental Animal Center of Soochow University

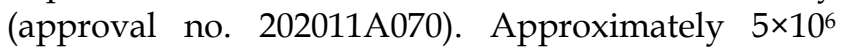
normal H1299 cells or stable YTHDC2-overexpressing H1299 cells were implanted subcutaneously into the 
right flank of the animals ( $\mathrm{n}=8$ mice per group). Animals were euthanized by cervical dislocation $\sim 30$ days after implantation, and tumors were collected and photographed. The maximum xenograft tumor size obtained in the study was $12 \mathrm{~mm}$.

\section{IHC}

Tumor samples obtained from xenograft model were fixed with $4 \%$ paraformaldehyde, dehydrated through a graded series of ethanol and embedded in paraffin. Sections (3- $\mu \mathrm{m}$ thick) were deparaffinized, rehydrated, and stained with hematoxylin and eosin. For IHC, the tissue sections were blocked with 5\% goat serum and incubated with primary antibodies at $4{ }^{\circ} \mathrm{C}$ overnight. Next, the sections were incubated with a goat anti-rabbit secondary antibody for $20 \mathrm{~min}$ at room temperature, and then subjected to streptavidinHRP staining for $30 \mathrm{~min}$. After staining with diaminobenzidine, the sections were stained with hematoxylin and subjected to dehydration. The primary antibodies used for IHC assay were purchased from Abcam (anti-YTHDC2) and ProteinTech Group, Inc. (anti-Ki67, anti-cyclin D1, anti-CDH1, anti-CDH2, anti-CYLD and anti-NFкBp65).

\section{Copy number analysis}

In order to explore the association between continuous YTHDC2 mRNA expression and discrete copy number status, patients with LUAD and LUSC were distributed into 5 subgroups (homozygous deletion, single copy deletion, normal diploid copy, low-level copy number amplification and high-level copy number amplification) [41]. In order to obtain the prognostic significance, the prognostic significance and the correlation between DNA copy number and smoking history were analyzed. The copy numbers of YTHDC2 in patients with lung cancer were also analyzed using the Oncomine database (https://www.oncomine.org/resource/ login.html), which is an online platform used for genome-wide data-mining [42] with the following parameters: The threshold P-value was set at 0.001 with a minimum 2-fold change. Data cohorts that had prominent copy number loss in NSCLC compared with that in normal tissue were extracted for analysis.

\section{TaqMan copy number assay}

Total genomic DNA of CS-exposed cells and lung cancer cell lines were isolated using a genomic DNA isolation kit (Tiangen Biotech Co., Ltd.). TaqMan copy number assays for YTHDC2 (probe ID: Hs06075146_cn) were performed according to the manufacturer's instructions. In total, 20 ng genomic DNA was mixed with $1 \mu$ YTHDC2 copy number assay (cat. no. 4400291; Thermo Fisher Scientific, Inc.),
$1 \mu l$ copy number reference assay (cat. no. 4403326; Thermo Fisher Scientific, Inc.) and $10 \mu l$ Master Mix (cat. no. A30865; Thermo Fisher Scientific, Inc.) to a final volume of $20 \mu \mathrm{l}$. The reference gene in the copy number reference assay was RNase $\mathrm{P}$ labeled with VIC dye, which is known to have two copies in a diploid genome. Copy number PCR was performed with a QuantStudio 6 Flex Real-Time PCR system (Thermo Fisher Scientific, Inc.) and further analyzed with CopyCaller v2.1 (Thermo Fisher Scientific, Inc.) following the manufacturer's instructions.

\section{RNA immunoprecipitation (RIP) sequencing}

RIP-RNA high-throughput sequencing was performed by Shanghai Cloud-seq Biotech Co.,Ltd. RIP was performed as previously described [43]. RNA was extracted using TRIzol ${ }^{\circledR}$ and ribosomal RNAs (rRNAs) were removed using NEBNext rRNA Depletion Kit (New England BioLabs, Inc.). RNA libraries were constructed using rRNA-depleted RNAs with NEBNext ${ }^{\circledR}$ Ultra $^{\mathrm{TM}}$ II Directional RNA Library Prep Kit (New England BioLabs, Inc.) according to the manufacturer's instructions. Libraries were controlled for quality and quantified using the BioAnalyzer 2100 system (Agilent Technologies, Inc.). Library sequencing was performed on an Illumina HiSeq instrument with 150-bp paired end reads.

\section{RNA decay assay}

RNA decay assay was performed as previously described [44]. H1299 cells transfected with pYTHDC2 or blank vector were seeded on 6-well plates, and actinomycin D (Hangzhou Fude Biological Technology Co. LTD) at a final concentration of 10 $\mu \mathrm{g} / \mathrm{ml}$ was added to the medium. Cells were collected at $0,1,2,3,4$ and $5 \mathrm{~h}$ after addition of actinomycin D. RNA was extracted and quantified by RT-qPCR. The same aforementioned primers were used to detect CYLD mRNA levels. Ct values were normalized to the $\mathrm{Ct}$ value of $\mathrm{t}=0$ to obtain $\Delta \mathrm{Ct}$ value $(\Delta \mathrm{Ct}=$ Mean $\mathrm{Ct}$ of each time point - Mean $\mathrm{Ct}$ of $\mathrm{t}=0$ ), and the relative abundance for each time point were calculated $\left(\right.$ mRNA abundance $\left.=2^{-\Delta \mathrm{CT}}\right)$.

\section{Electrophoretic mobility shift assay (EMSA)}

Nuclear proteins from H1299 cells were isolated using Nuclear and Cytoplasmic Protein Extraction kit (CWBio) according to the manufacturer's protocol. Protein concentration was measured using an enhanced BCA protein assay kit (Beyotime Institute of Biotechnology). Biotin-labeled NF-KB consensus oligonucleotides were obtained from Beyotime Institute of Biotechnology. Nuclear extracts $(5 \mu \mathrm{g})$ were added to $20 \mu \mathrm{l}$ binding reactions and incubated for $20 \mathrm{~min}$ at room temperature. EMSA was 
performed with Chemiluminescent EMSA kit (Beyotime Institute of Biotechnology) according to the manufacturer's protocol.

\section{m6A methylated RIP (meRIP)}

The $\mathrm{m}^{6} \mathrm{~A}$ distribution in the CYLD mRNA sequence was predicted using the sequence-based RNA adenosine methylation site predictor (SRAMP) algorithm [45]. Specific primers were designed for meRIP analysis according to the high-confidence fragments. Magna MeRIP m6 A kit (cat. no. 17-10499; MilliporeSigma) was used to analyze the $\mathrm{m}^{6} \mathrm{~A}$ modification in H1299 cells according to the manufacturer's recommendations. In brief, $150 \mu \mathrm{g}$ total RNA was isolated and randomly fragmented into fragments of $\sim 100 \mathrm{bp}$. RNA samples were then immunoprecipitated with anti- $\mathrm{m}^{6} \mathrm{~A}$ or anti-mouse IgG antibody-coated magnetic beads. The $\mathrm{m}^{6} \mathrm{~A}$-modified RNA fragments were eluted and analyzed by PCR and RT-qPCR. Relative enrichment of $\mathrm{m}^{6} \mathrm{~A}$ was normalized to the input.

\section{Statistical analysis}

GraphPad Prism v7.0 software (GraphPad Software, Inc.) was used for all statistical analyses. Values are presented as the mean \pm standard deviation $(n=3)$. Student's unpaired t-test was used to detect the statistical differences in expression of YTHDC2 between tumor and adjacent non-tumor tissues, as well as between clinicopathological features and smoking history. Kaplan-Meier survival curves and Cox regression analysis were performed using the R survival package. Pearson's correlation coefficients were calculated to evaluate the association between DNA copy number and YTHDC2 expression using $\mathrm{R}$ software (Version 4.0.4). $P<0.05$ was considered to indicate a statistically significant difference.

\section{Results}

\section{Expression and clinical significance of YTHDC2 in lung cancer}

According to the GEPIA online tool database, the expression levels of YTHDC2 were observed to be different in different types of cancer. Compared with those in normal tissues, YTHDC2 expression levels were significantly downregulated in 7 types of cancer, including LUAD and LUSC (Figure 1A), ovarian serous cystadenocarcinoma, testicular germ cell tumor, thyroid carcinoma, uterine corpus endometrial carcinoma and uterine carcinosarcoma, while they were increased in lymphoid neoplasm diffuse large B-cell lymphoma and thymoma (Figure S1). The mRNA expression of YTHDC2 in lung cancer tissues was less than that in normal adjacent tissues in two
GEO datasets, GSE32665 (Figure 1B) and GSE19188 (Figure 1C). The IHC results of lung cancer tissue arrays showed a significantly lower YTHDC2 expression in lung cancer tissues than that in normal tissues (Figure 1D and E, S2 and S3). Based on the data of $\mathrm{HPA}, 50 \%$ of the lung cancer tissues showed no staining for anti-YTHDC2 antibody, while the 3 normal tissues showed low staining (Figure S4). The present IHC results showed decreased YTHDC2 protein expression in tumor tissues with larger diameters (maximum diameter $\geq 5 \mathrm{~cm}$ ), or at advanced $\mathrm{T}$ stages (T3 and T4) or pathological stages (Figure 1F-H).

When the pathological stage is considered, the expression of YTHDC2 mRNA in stage II lung cancer tissues in the TCGA LUAD and GSE41271 cohorts was significantly lower than that in stage I (Figure S4A and C). The mRNA expression of YTHDC2 in stage IV tumor tissues in the TCGA LUSC cohort was significantly lower than that in stage I (Figure S4B). For invasion depth $\mathrm{T}$ stage, the mRNA expression of YTHDC2 in lung cancer tissues with T3 or/and T4 stages in the TCGA LUAD, TCGA LUSC and GSE30219 cohorts were also significantly lower than those in lung cancer tissues with T1 stage (Figure S4D-F). In addition, the expression of YTHDC2 in M1 lung cancer was significantly lower than that in M0 lung cancer in the TCGA LUSC cohort (Figure S4H). Similarly, for lymph node metastasis $\mathrm{N}$ stage, the expression of YTHDC2 in N1-stage lung cancer tissues in the TCGA LUAD and GSE30219 cohorts was significantly lower than that in N0 stage tissues, and the expression of YTHDC2 in N2 stage lung cancer tissues in the GSE30219 cohort was also significantly lower than that in N0 stage tissues (Figure S4J-L). To identify the association between YTHDC2 expression and smoking history, the present study analyzed the differential expression of YTHDC2 mRNA in patients with lung cancer with different smoking histories. For the TCGA LUAD cohort, the YTHDC2 mRNA expression level was low in current smokers and reformed smokers for $\leq 15$ years compared with that in individuals who had never smoked. Higher YTHDC2 mRNA expression level was found in reformed smokers for $>15$ years compared with that in current smokers (Figure S4M). YTHDC2 mRNA expression in smoking patients was remarkedly lower than that of non-smokers in the GSE41271 cohort (Figure S4O).

Next, the present study evaluated whether YTHDC2 was downregulated in our established CS-induced carcinogenic model [10]. RT-qPCR and western blotting revealed that YTHDC2 was significantly downregulated in CS-exposed cells (S10, S20 and S30) at the mRNA and protein levels compared with that in unexposed BEAS-2B cells. The 
expression levels were found to be dependent on smoke-exposure time (Figure 1I-K).

As shown in Figure S5, univariate Cox model and Kaplan-Meier survival analysis revealed that increased YTHDC2 mRNA expression was significantly associated with improved survival $(\mathrm{HR}<1, \mathrm{P}<0.05)$ in GSE41271, GSE30219 and TCGA lung cancer datasets.

\section{YTHDC2 functional prediction by correlation and proteomic analyses}

Based on the analysis of the LinkedOmics online tool, 1,037 and 724 genes, respectively, were positively correlated with low YTHDC2 expression [correlation coefficient $(\mathrm{r})>0.3, \mathrm{P}<0.001]$ in LUAD and LUSC, while 648 and 147 genes were negatively correlated ( $\mathrm{r}$ $<-0.3, \mathrm{P}<0.001$ ), respectively (Figure S6A-B). Further

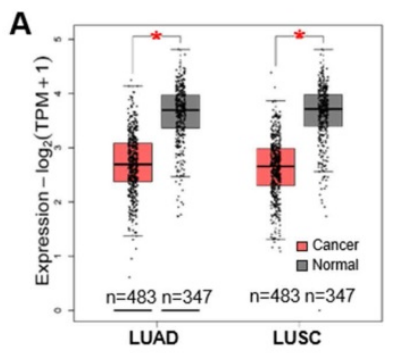

B
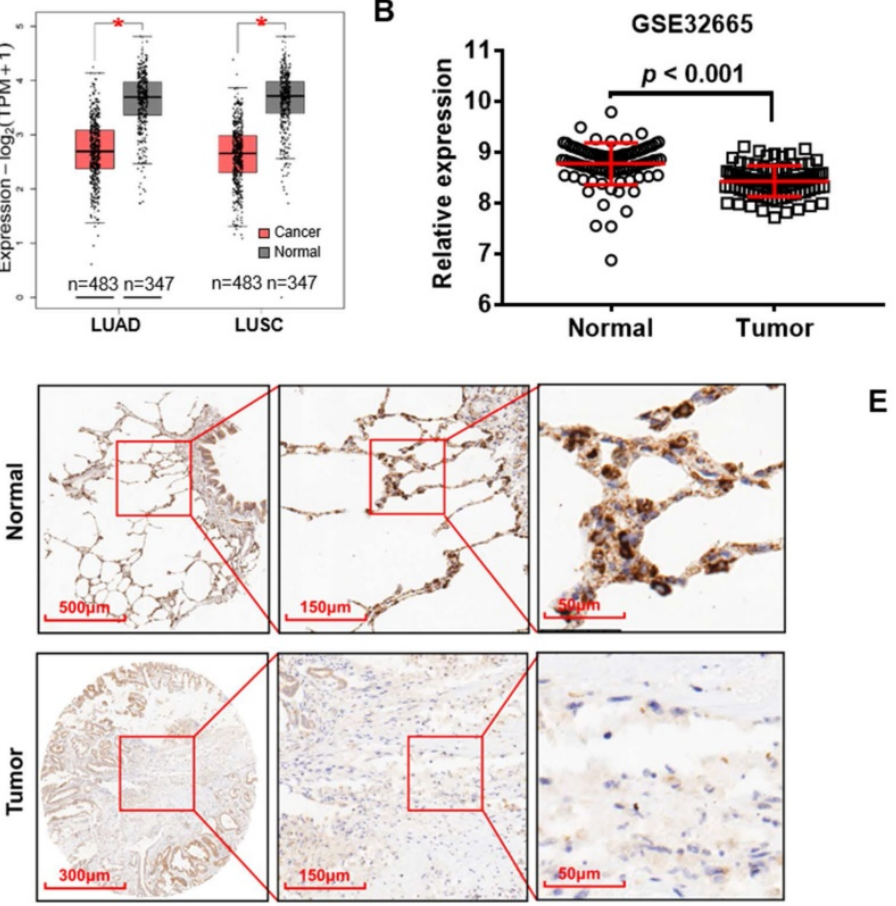

$\mathrm{F}$

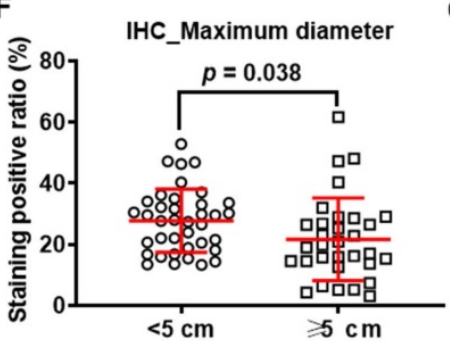

G

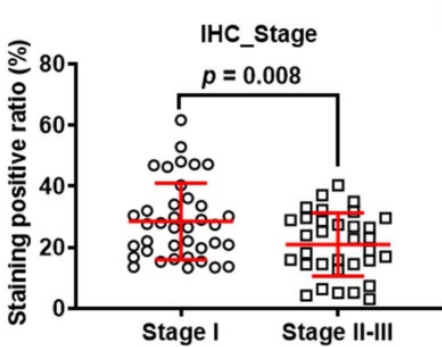

$\mathbf{J}$

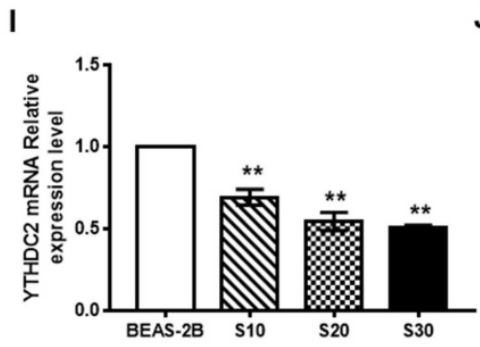

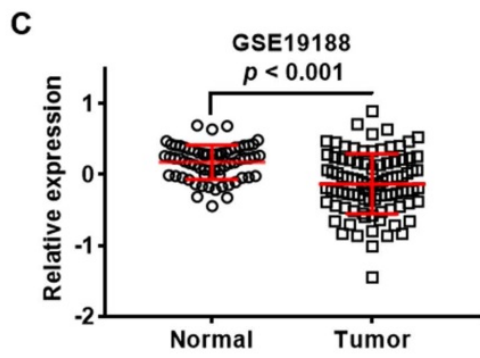

E

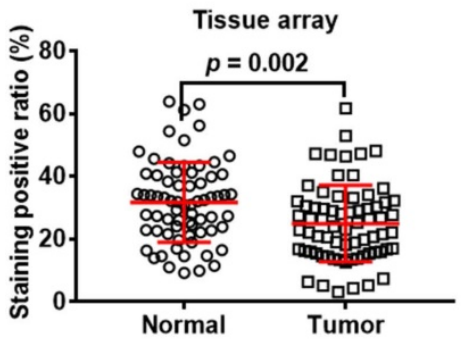

H

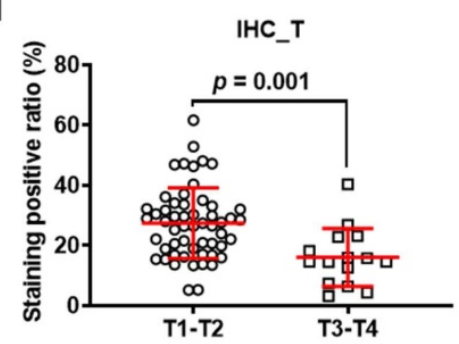

K

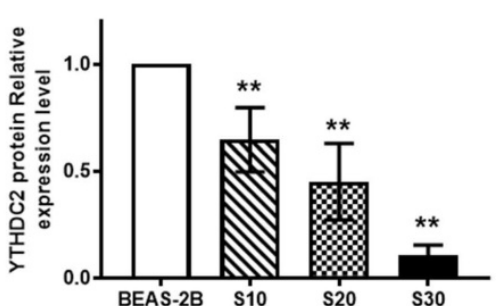

Figure 1. YTHDC2 gene and protein expression is downregulated in patients with lung cancer from The Cancer Gene Atlas, Gene Expression Omnibus and Human Protein Atlas databases, as well as in CS-exposed cells. (A) Differential analysis of YTHDC2 mRNA expression in lung cancer tissues based on the Gene Expression Profiling Interactive Analysis tool. *P<0.05 vs. normal tissues. Differential analysis of YTHDC2 mRNA expression in lung cancer tissues from (B) GSE32665 and (C) GSE19188 datasets. (D) Representative IHC images showed that YTHDC2 staining was found in the cell cytoplasm in lung cancer and normal lung tissues. High expression of YTHDC2 could be found in adjacent normal tissues, while its expression was decreased in the majority of lung cancer tissues. (E) Differential analysis of YTHDC2 staining positive ratio quantitated by IHC Profiler in lung cancer tissue arrays. YTHDC2 staining positive ratio in lung cancer tissues with different (F) maximum diameter, (G) pathological stage and (H) invasion depth. (I) Relative mRNA expression level of YTHDC2 in CS-exposed cells (S10, S20 and S30) and normal BEAS-2B cells. Western blot analysis (l) and quantitative results (K) of YTHDC2 protein expression in CS-exposed cells (S10, S20 and S30) and normal BEAS-2B cells. S10, S20 and S30 represent BEAS-2B cells exposed to CS for 10,20 and 30 passages, respectively. **P $<0.01$ vs. normal BEAS-2B cells. IHC, immunohistochemistry; YTHDC2, YTH domain containing 2; CS, cigarette smoke. 
Kyoto Encyclopedia of Genes and Genomes (KEGG) enrichment analysis showed that these YTHDC2related genes in LUAD were significantly enriched in 'RNA transport', 'mTOR signaling pathway', 'cell cycle' and 'RNA degradation' (Figure 2A), while the YTHDC2-related genes in LUSC were significantly enriched in; RNA transport' and 'RNA degradation' (Figure 2B). For biological process enrichment, the YTHDC2-related genes in LUAD and LUSC were also enriched in several cancer-related processes, including 'NF- $\mathrm{BB}$ signaling', 'mRNA splicing', 'cell cycle', 'cell division', 'TNF-mediated signaling' and 'Wnt signaling pathway' (Figure S6C-D). TSGs and MRGs were obtained from the TSGene and HCMDB databases. A total of 58 and 17 TSGs was positively associated with YTHDC2 in LUAD (Figure 2C) and LUSC (Figure 2D), respectively. A total of 62 and 12 MRGs were negatively correlated with YTHDC2 in LUAD (Figure S6I) and LUSC (Figure S6J), while 89 and 31 MRGs were positively correlated with YTHDC2 in LUAD (Figure S6I) and LUSC (Figure S6J), respectively. Among these positively correlated TSGs and MRGs, adenomatous polyposis coli, chromodomain helicase DNA binding protein 1 and enhancer of zeste homolog 2 were found to be significantly reduced in CS-exposed cells (S10, S20 and S30) and lung cancer cells (A549 and H1299) (Figure S6K-M).

In addition, among the quantified differentially expressed proteins (DEPs) identified by TMT proteomics profiling, 487 were upregulated and 333 were downregulated (Figure 2E and Table S1). Based on the subcellular location annotation, certain DEPs were found $\geq 1$ locations within the cell: In total, $36.66 \%$ were in the cytoplasm, $27.89 \%$ in the nucleus, $11.57 \%$ in mitochondria, $9.74 \%$ in the extracellular matrix and $5.60 \%$ in the plasma membrane (Figure 2F). To assess the potential biological function of YTHDC2, DEPs were subjected to GO functional and KEGG pathway enrichment. According to biological process classification, these DEPs were involved in various cellular processes, including 'translational initiation', 'RNA splicing', 'response to hypoxia', 'regulation of RNA stability' and 'cell cycle process and apoptotic signaling' (Figure 2G). Regarding KEGG pathway enrichment, these DEPs were enriched in several important pathways, including 'spliceosome', 'ribosome', 'proteasome', 'citrate cycle' and 'endocytosis' (Figure 2H). Among the 820 DEPs, 264 proteins were depicted in the STRING database to identify several clusters by plug-in Molecular Complex Detection (MCODE) in Cytoscape (Figure 2I). The DEPs in the top 3 clusters were imported into the DAVID database for biological process enrichment. The cluster 1 sub-network was related to
'RNA splicing', 'mRNA processing' and 'regulation of RNA stability'; cluster 2 sub-network was linked to 'non-coding RNA (ncRNA) localization', 'RNA localization' and 'ribosome biogenesis'; and cluster 3 was involved in the 'regulation of RNA stability', 'Wnt signaling pathway' and 'cell cycle phase transition' (Figure 2J-K).

\section{YTHDC2 downregulation increases the proliferation and migration of lung cancer cells}

In order to study the regulation of the YTHDC2 gene in cancer cell proliferation, siRNA (siYTHDC2) or expression plasmid vector (pYTHDC2) was transfected into normal human lung BEAS-2B cells, as well as in the lung cancer cell line H1299 to knockdown or overexpress YTHDC2. Data from flow cytometry analysis showed that knocking down YTHDC2 promoted cell cycle progression by accelerating the progression of cells from the $G_{1}$ to the $S$ phase in BEAS-2B and H1299 cells (Figure 3A, C). EdU assay showed that the proliferation of BEAS-2B and H1299 cells was significantly enhanced by knocking down YTHDC2 (Figure 3E). By contrast, the cell cycle progression and proliferation capacity of S30 and H1299 cells were markedly decreased by the overexpression of YTHDC2 (Figure 3B, D, F). The immunofluorescence results showed an elevated Ki67 expression in YTHDC2-knockdown cells and a reduced expression in YTHDC2-overexpressing cells (Figure 3G-J). RT-qPCR analysis revealed the upregulation of cyclin D1 expression, a protein required for cell cycle $G_{1} / S$ transition, in YTHDC2knockdown cells and a downregulation in YTHDC2-overexpressing cells (Figure 3K-L). These observations suggested that YTHDC2 is required for the cell cycle progression and proliferation of lung cancer cells.

Regarding cell migration ability, wound healing assays (Figure $\mathbf{4 A}$ and B) and Transwell assays (Figure 4C) also showed that the migration capacity of BEAS-2B and H1299 cells was significantly enhanced by knocking down YTHDC2. The results from RT-qPCR and western blotting revealed that the mRNA and protein levels of the mesenchymal marker $\mathrm{N}$-cadherin ( $\mathrm{CDH} 2)$ were significantly upregulated, while the epithelial marker E-cadherin (CDH1) was downregulated in siYTHDC2-transfected cells compared with the findings in the negative control group (Figure 4E). By contrast, $\mathrm{CDH} 2$ was significantly downregulated, while $C D H 1$ was upregulated, in PYTHDC2-transfected cells compared with the findings in the blank vector group (Figure $4 \mathrm{~F})$. In addition, the changes in $\mathrm{CDH} 1$ and $\mathrm{CDH} 2$ protein levels were consistent with the mRNA levels in both knockdown and overexpressing cells (Figure 
4G-J). These observations suggested that YTHDC2 is of lung cancer cells. required for the cellular migration and EMT process

A

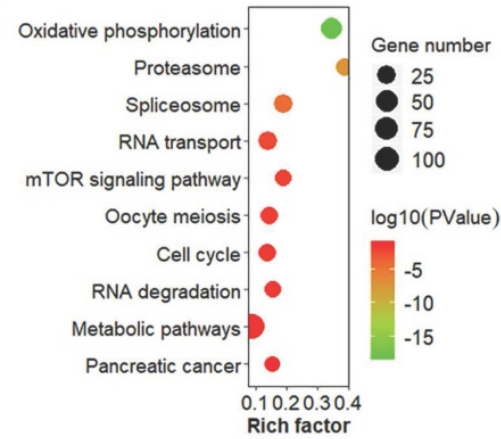

B

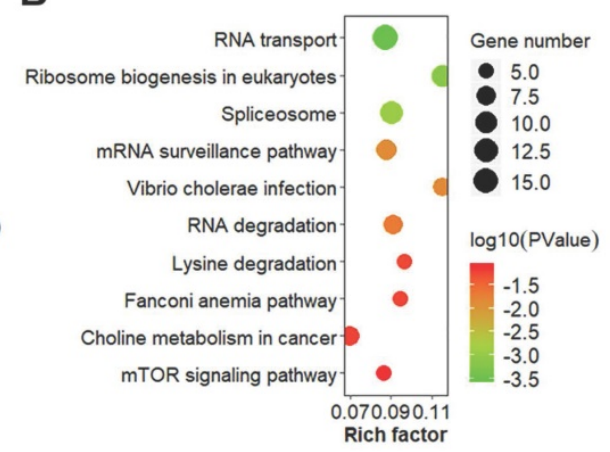

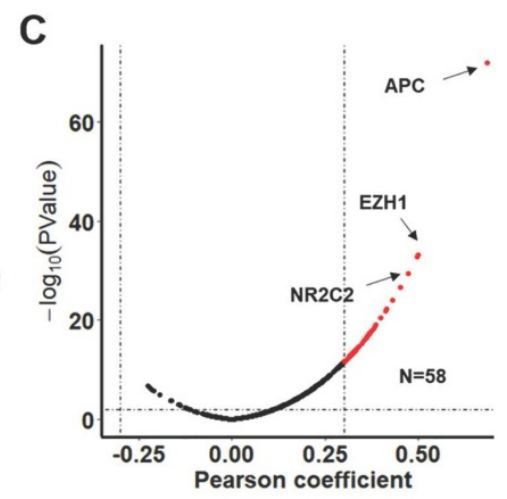

D

E

F
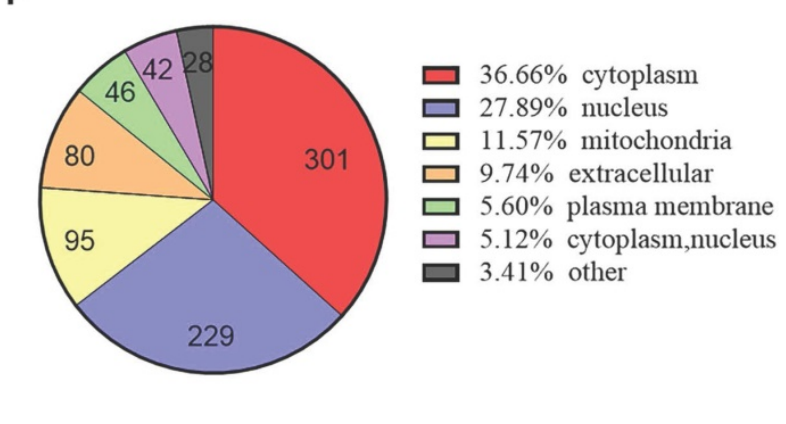

G
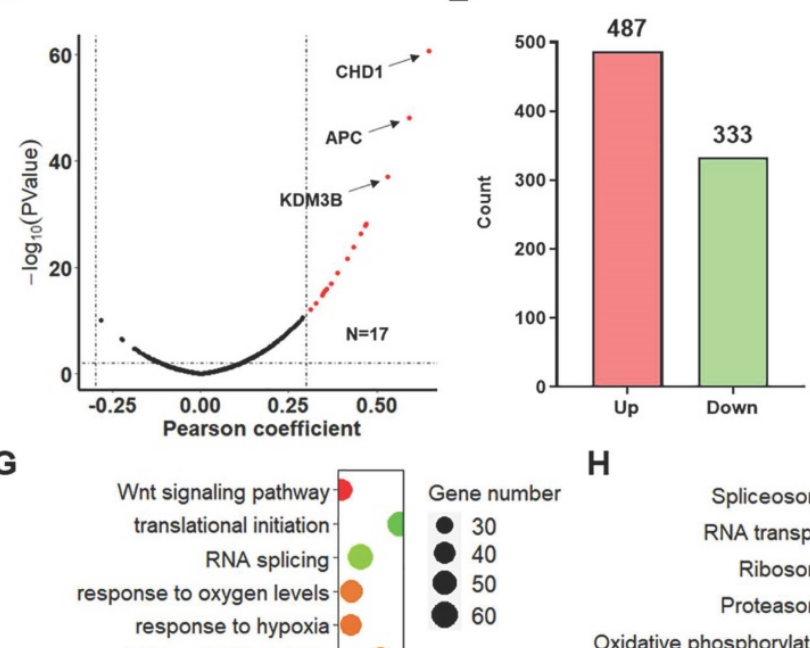

regulation of RNA stability -

regulation of cell cycle process-

NIKJNF-kappaB signaling-

cellular response to TNF-

apoptotic signaling pathway-

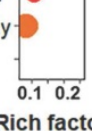

H

Spliceosome

RNA transport

Ribosome

Proteasome

Oxidative phosphorylation

NAFLD

nRNA surveillance pathway-

Endocytosis

Citrate cycle (TCA cycle)

Carbon metabolism

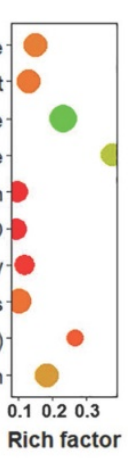

Gene number |

10
15
20
25
30
35

$\log 10$ (Pvalue)
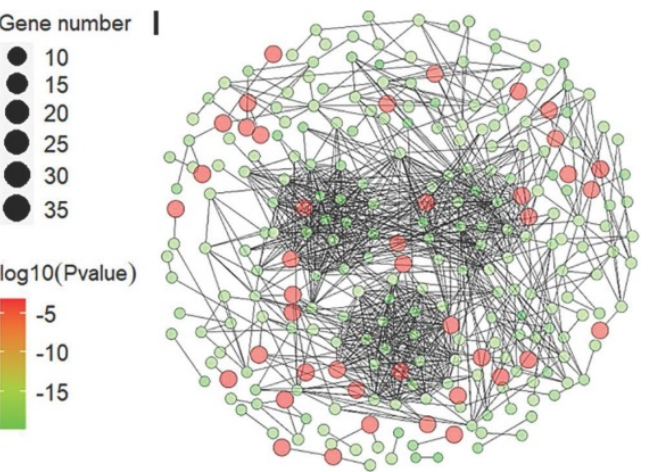

L
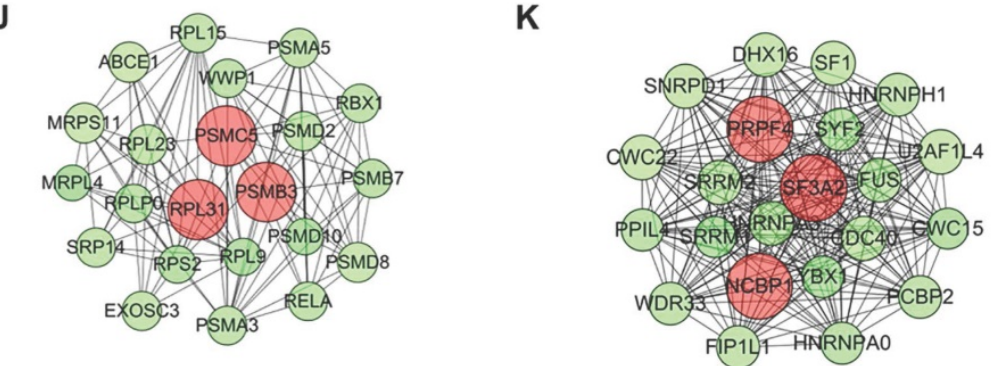

K

\begin{tabular}{lc}
\hline Description & $\begin{array}{l}\text { Log10 } \\
\text { (P value) }\end{array}$ \\
\hline regulation of RNA stability & -13.68 \\
Wnt signaling pathway & -9.66 \\
cell cycle phase transition & -8.90 \\
\hline
\end{tabular}

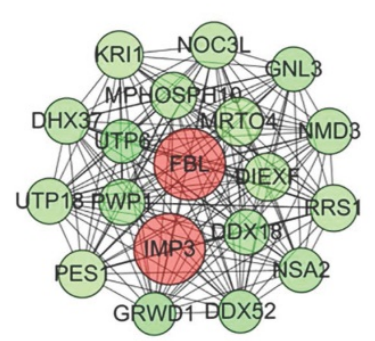

\begin{tabular}{lc}
\hline Description & $\begin{array}{l}\text { Log10 } \\
\text { (P value) }\end{array}$ \\
\hline ribosome biogenesis & -20.40 \\
ncRNA processing & -14.63 \\
RNA localization & -3.426
\end{tabular}

Figure 2. Proteomics analysis of YTHDC2-knockdown cells. Bubble chart showing the KEGG enrichment results of the genes associated with (A) LUAD and (B) LUSC. The larger the rich factor, the greater the degree of enrichment. The color gradient from red to green represents the P-value; the closer to green color, the lower the P-value and the higher the significance level corresponding to the enrichment. Volcano plots showing the tumor suppressor genes in YTHDC2-related genes in (C) LUAD and (D) LUSC. (E) Bar graph showing the number of upregulated and downregulated proteins in YTHDC2-knockdown cells. (F) Subcellular distribution of DEGs. (G) Biological process and (H) KEGG pathway analysis of DEGs. (I) The protein-protein interaction network of DEGs was constructed using Cytoscape. (J-L) Identification of the top 3 significant clusters using the plug-in Minimal Common Oncology Data Elements in Cytoscape, and biological process enrichment analysis of the proteins in these clusters. KEGG, Kyoto Encyclopedia of Genes and Genomes; YTHDC2, YTH domain containing 2; LUSC, lung squamous cell carcinoma; LUAD, lung adenocarcinoma; DEGs, differentially expressed genes. 
A
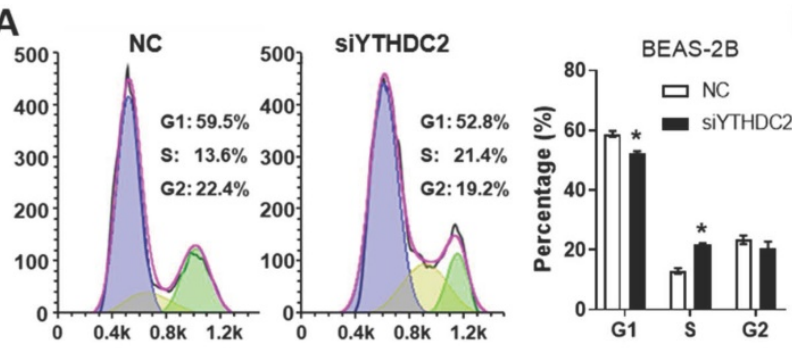

B
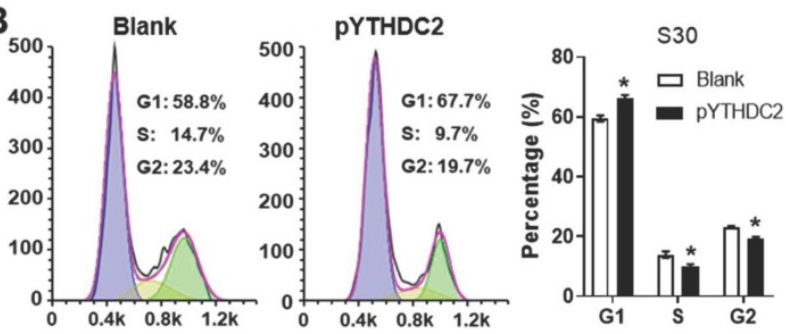

C
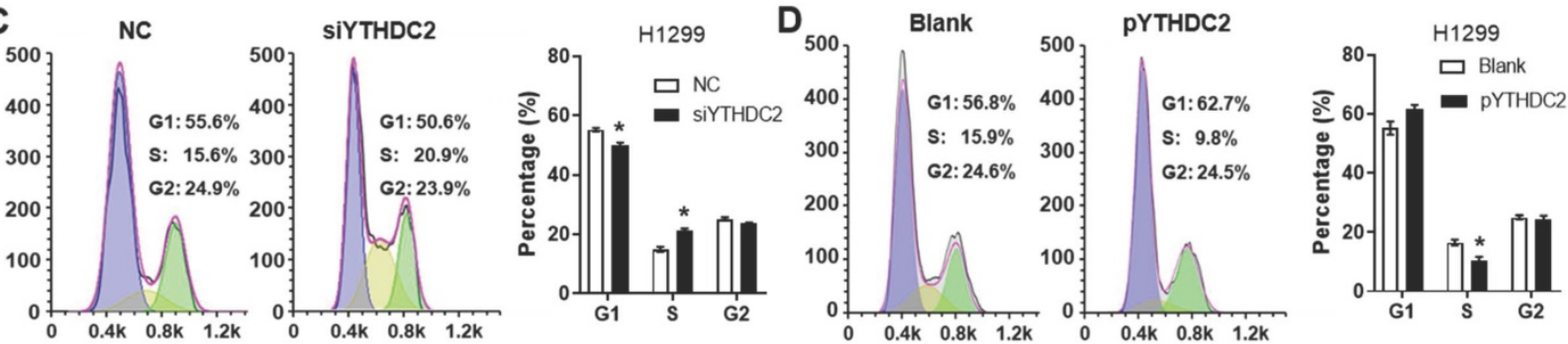

E

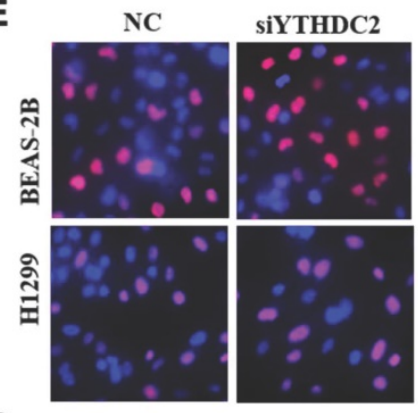

G

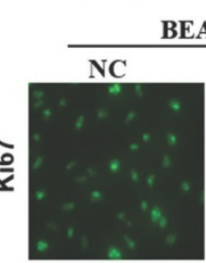

SiYTHDC2
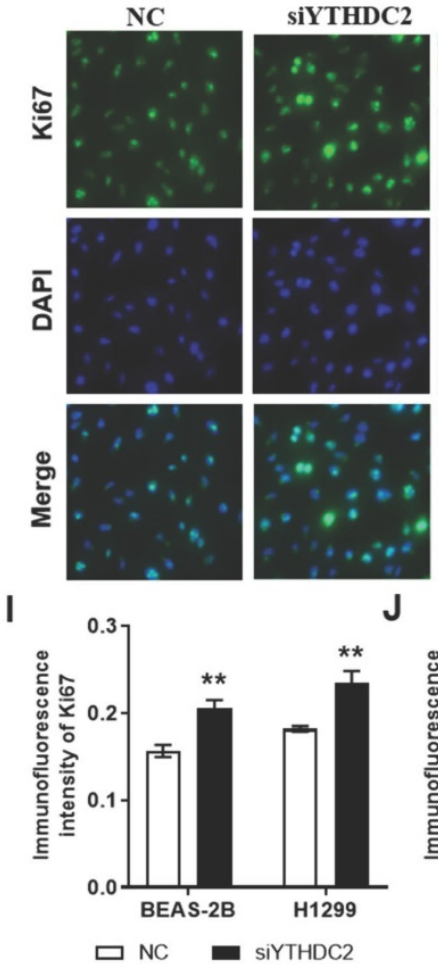

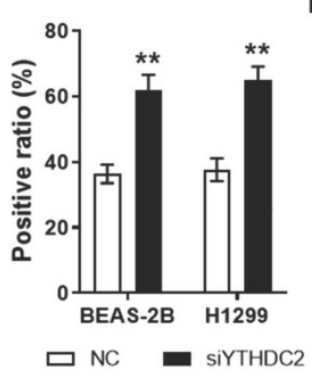

$\mathbf{F}$
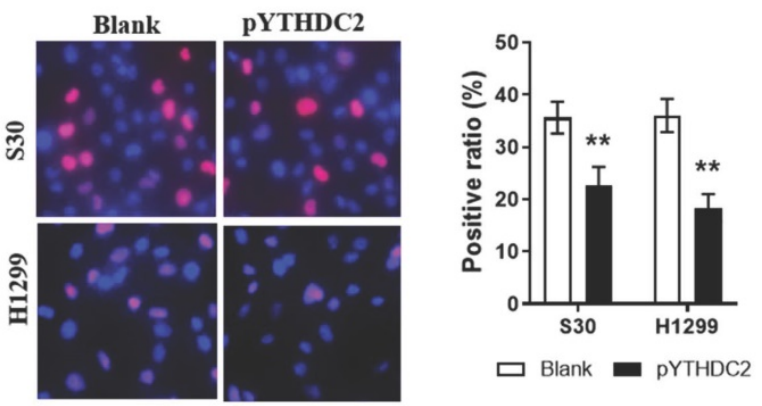

H
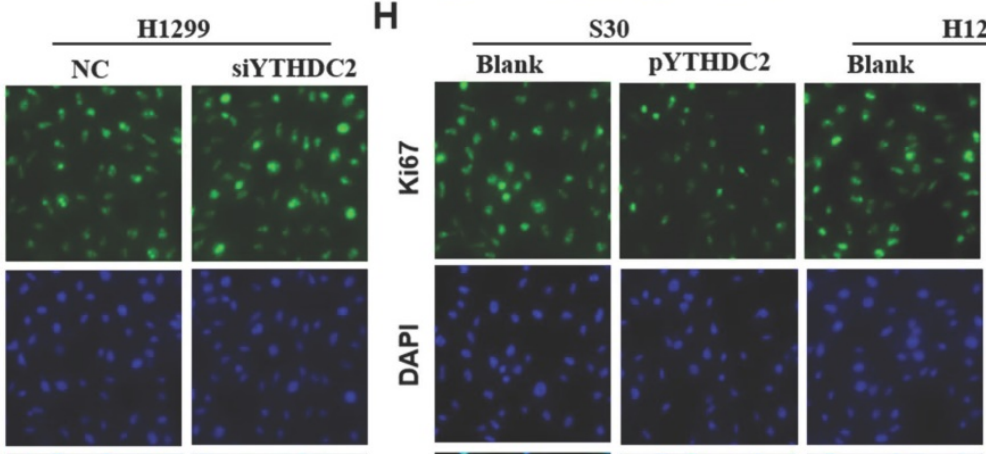

pYTHDC
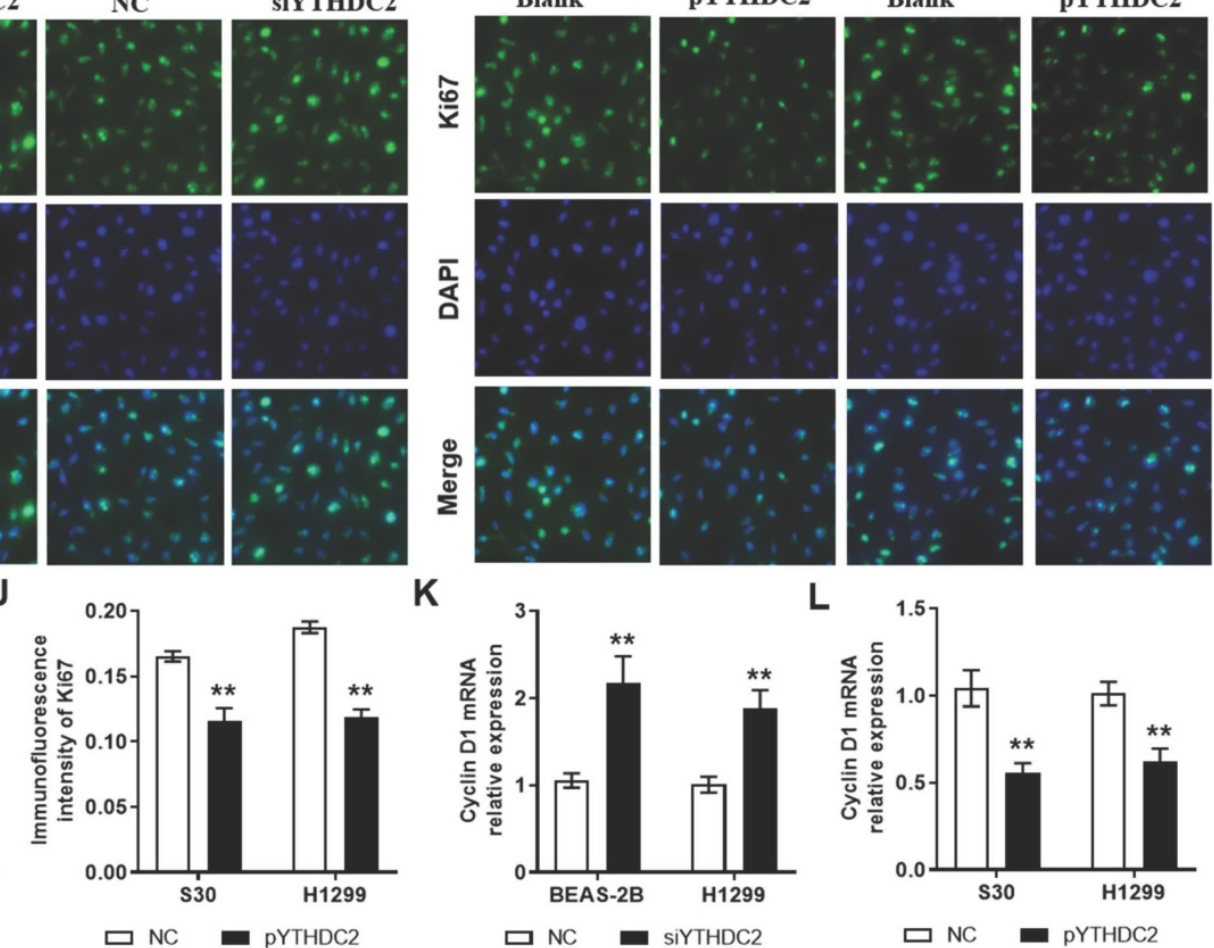

K
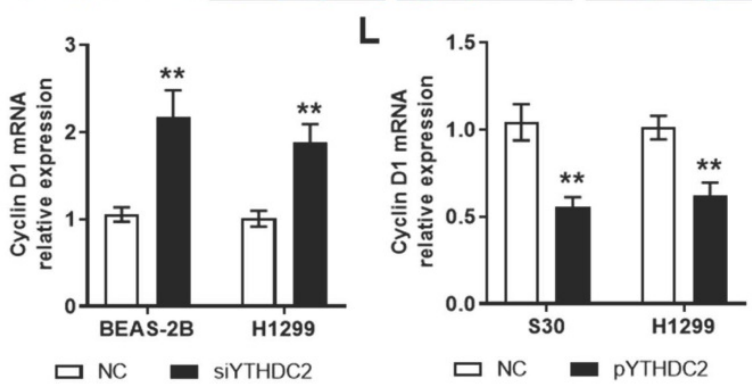

Figure 3. YTHDC2 downregulation promotes lung cancer cell proliferation. Representative images and quantification results of cell cycle of (A) BEAS-2B and (C) H1299 cells transfected with siYTHDC2 and NC. Representative images and quantification results of cell cycle of (B) S30 and (D) H1299 cells transfected with overexpression vector (pYTHDC2) and blank vector. (E) Cell proliferation was measured in BEAS-2B and H1299 cells transfected with siYTHDC2 and NC by EdU cell proliferation assay. (F) Cell proliferation were evaluated in S30 and H1299 cells transfected with pYTHDC2 and blank control by EdU cell proliferation assay. (G) Representative immunofluorescence images and (I) quantification results of Ki67 in BEAS-2B and H1299 cells transfected with siYTHDC2 and NC. (H) Representative immunofluorescence images and (J) quantification results of Ki67 in S30 and $\mathrm{H} 1299$ cells transfected with PYTHDC2 and blank control. Relative mRNA expression level of cyclin D1 in (K) BEAS-2B and H1299 cells transfected with siYTHDC2 and NC, as well as in (L) S30 and H1299 cells transfected with PYTHDC2 and blank control. *P<0.05 vs. NC or blank control group, * $* 0.01$ vs. NC or blank control group. YTHDC2, YTH domain containing 2; siRNA, small interfering RNA; NC, negative control; EdU, 5-ethynyl-2'-deoxyuridine. 
A

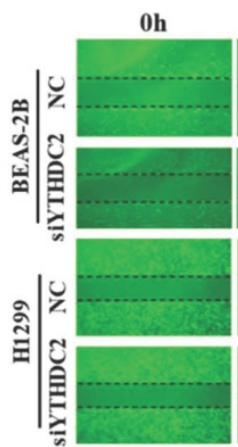

B
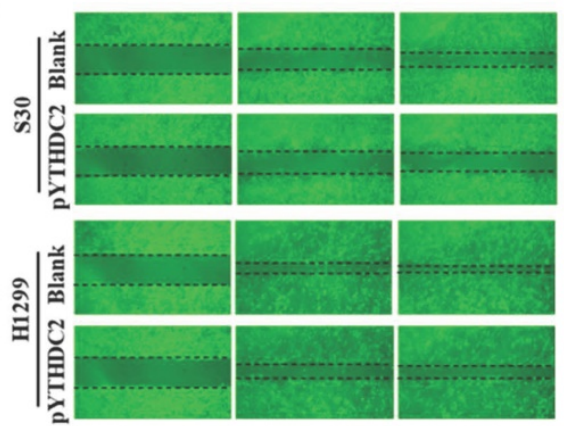

C
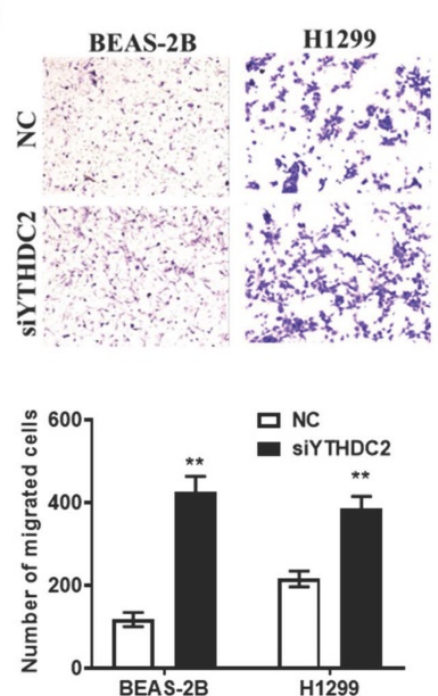

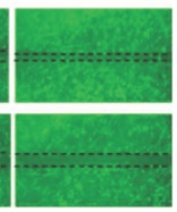

$48 \mathrm{~h}$
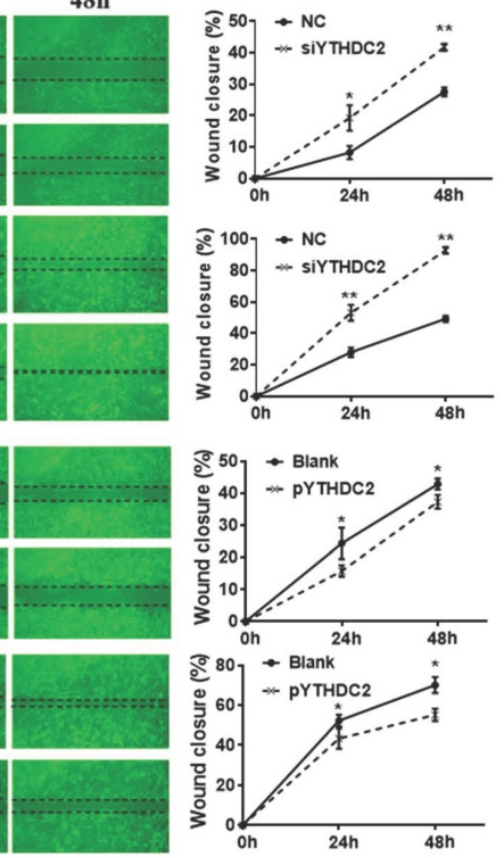

D
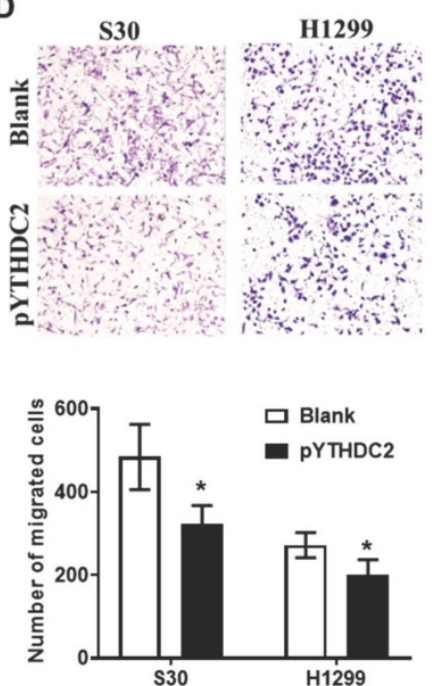

E

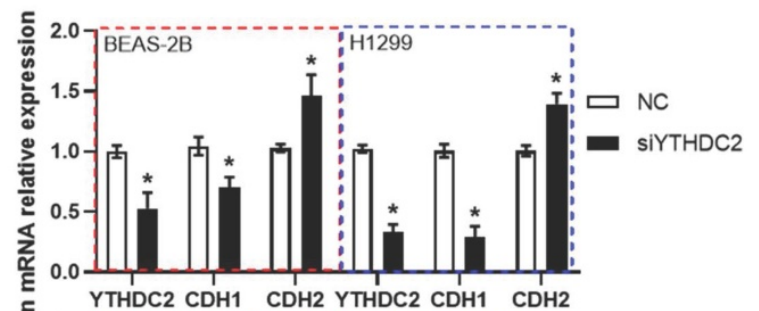

$F$

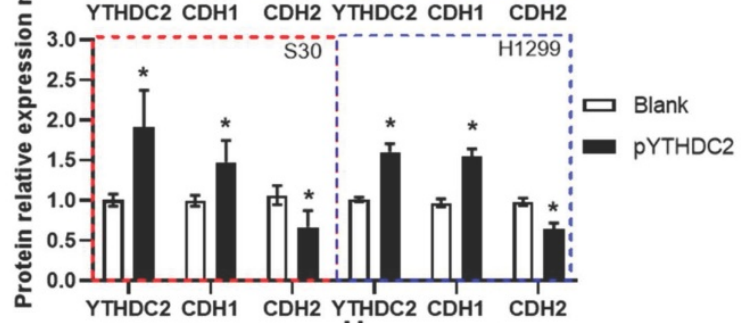

G
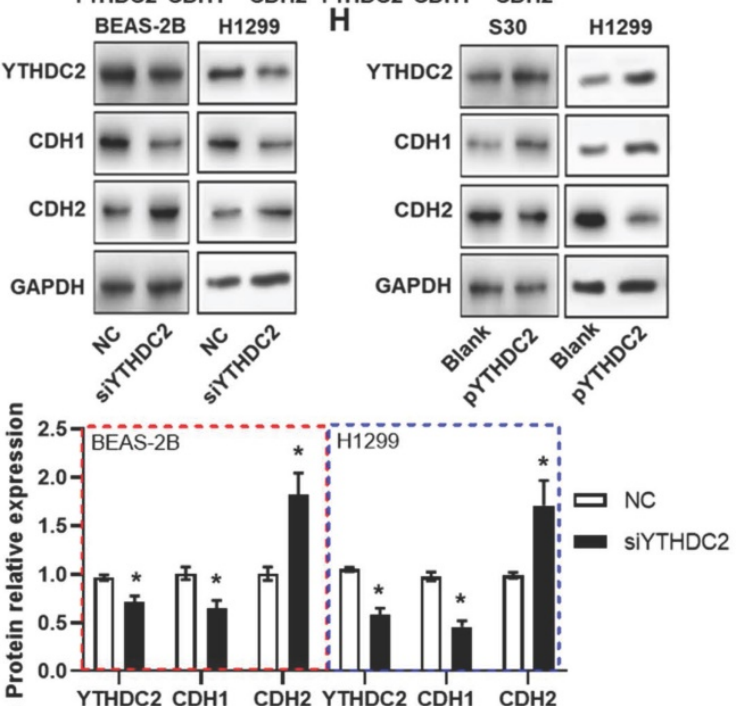

J

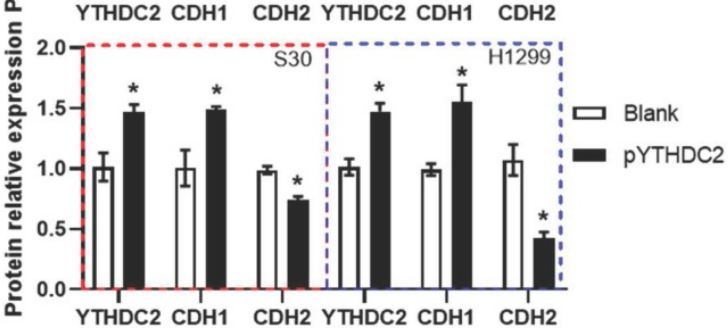

Figure 4. YTHDC2 downregulation promotes lung cancer cell migration. (A) Representative images and quantification of the wound healing assay showing that cell migration was significantly increased at 24 and $48 \mathrm{~h}$ after transfection with siYTHDC2 in BEAS-2B and H1299 cells, as well as following transfection with (B) the overexpressing vector PYTHDC2 and a blank vector. Representative images and quantification of the Transwell migration assay of BEAS-2B and H1299 cells transfected with (C) siYTHDC2 and (D) PYTHDC2. (E) Quantitative PCR analysis of CDH1 and CDH2 in normal BEAS-2B andH1299 cells transfected with siYTHDC2, (F) as well as in S30 and H1299 cells transfected with PYTHDC2. (G) Western blot analysis and (I) quantitative results of EMT markers in BEAS-2B and HI299 cells transfected with siYTHDC2. (H) Western blot analysis and () quantitative results of EMT markers in $\mathrm{S} 30$ and $\mathrm{H} 1299$ cells transfected with $\mathrm{PYTHDC2}$. ${ }^{*}<0.05$ vs. NC (blank vector) group, **P<0.01 vs. NC (blank vector) group. YTHDC2, YTH domain containing 2; siRNA, small interfering RNA; EMT, epithelial-mesenchymal transition; NC: negative control; $\mathrm{CDH}$ : E-cadherin; $\mathrm{CDH}$ 2: $\mathrm{N}$-cadherin.

\section{YTHDC2 upregulation suppresses lung cancer cell tumorigenesis in vivo}

To further validate the effect of YTHDC2 on lung cancer cell tumorigenesis in vivo, a xenograft animal model was established by subcutaneously injecting YTHDC2-overexpressing cells into the right armpits of BALB/c nude mice. As expected, YTHDC2 overexpression markedly attenuated the growth of tumors in nude mice compared with the findings in the blank group (Figure 5A and B). Similarly, the mean weight of the xenograft tumors in the pYTHDC2 group was also markedly lower than that in the blank group (Figure 5C). To further confirm the inhibitory effect of YTHDC2 on cell proliferation that was found in vitro, tumor tissues were digested with trypsin to form a single-cell suspension, and the cell cycle was then detected by flow cytometry. The results suggested a stronger cell proliferation ability in the pYTHDC2 group than in the blank group (Figure 5D and E). In addition, a significantly lower proportion of Ki67 and cyclin D1-positive cells in 
xenograft tumors derived from the pYTHDC2 group compared with that of the blank group was observed by IHC analysis (Figure 5F). The IHC results indicated that $\mathrm{CDH} 1$ was upregulated, while $\mathrm{CDH} 2$ was downregulated, in the xenograft tumors of the pYTHDC2 group compared with the findings in the blank group (Figure 5F).

\section{Reduced YTHDC2 expression is associated with gene copy loss in DNA}

The present study further explored the mechanisms of YTHDC2 dysregulation using the multi-omics data of lung cancer from TCGA database. A total of 509 patients with LUAD and 498 patients with LUSC had DNA amplification and mRNA expression measured simultaneously, and $39.92 \%$ of LUAD samples and $77.11 \%$ of LUSC samples showed low-level amplification (Figure 6A and D). Significant differences were observed in different DNA amplification groups in both lung cancer subtypes (Figure 6B and E). Further correlation analysis indicated that YTHDC2 mRNA expression values were significantly correlated with copy number values in LUAD and LUSC (Pearson correlation coefficient $=0.62$ and 0.44 , respectively; Figure $6 \mathrm{C}$ and F). In addition, 5 Oncomine datasets were extracted to verify the YTHDC2 copy numbers in lung cancer, and a significantly decreased copy number was found (Figure 6G).
A
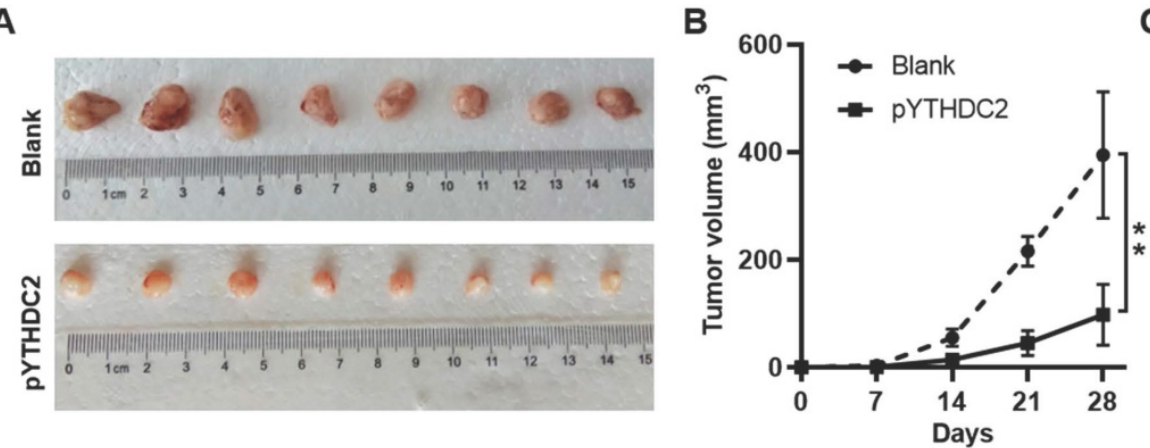

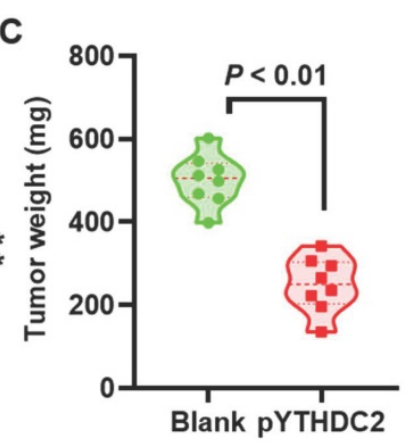

E

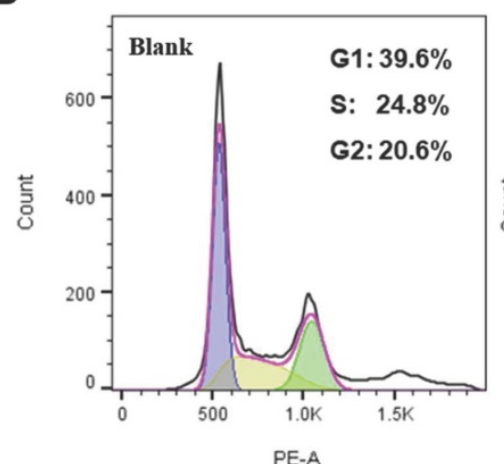

$\mathbf{F}$
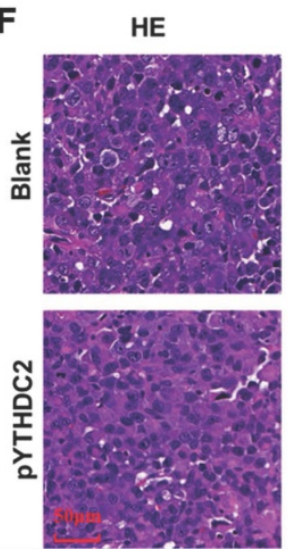

YTHDC2
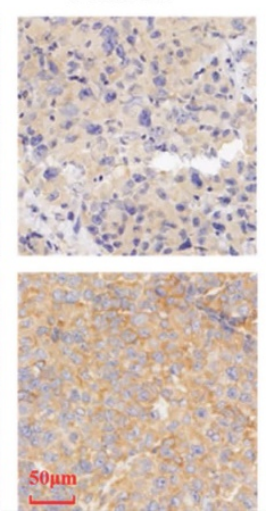

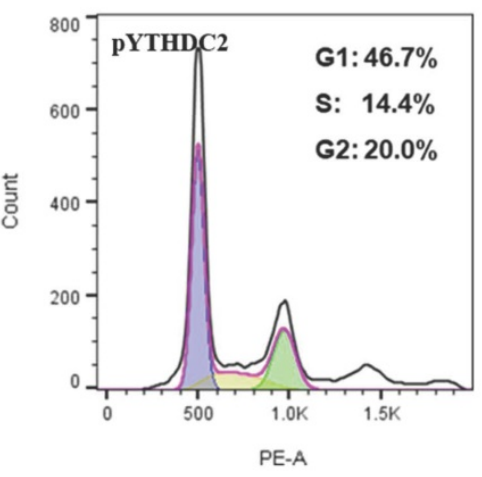

ki67

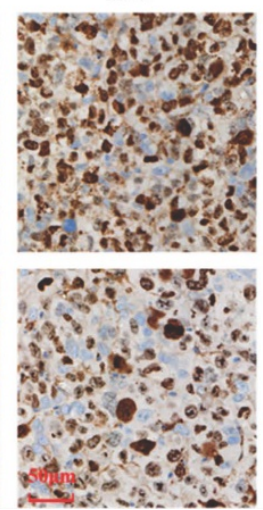

Cyclin D1
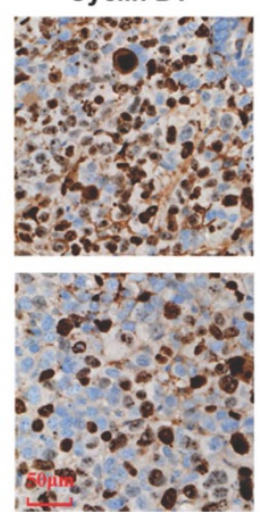

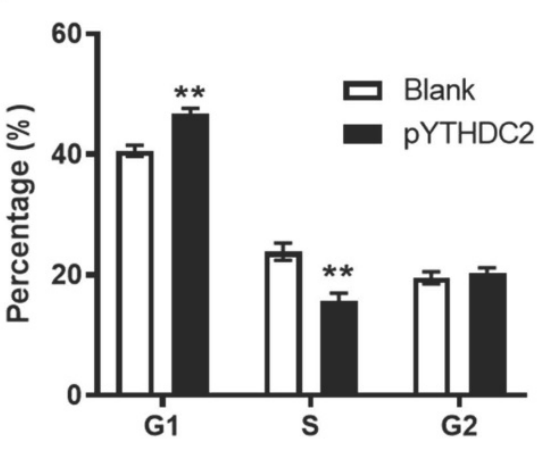

CDH1

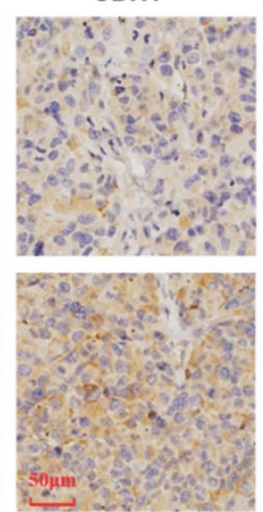

CDH2

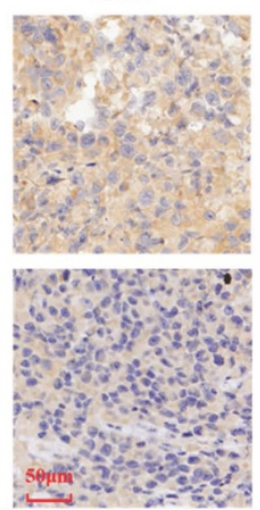

Figure 5. YTHDC2 overexpression suppresses H1299 cells growth in vivo. (A) Images of the xenograft tumors formed in nude mice injected with YTHDC2-overexpressing and control cells. (B) Volume and (C) weight of xenograft tumors isolated from nude mice. (D) Representative images and (E) quantification of the results of cell cycle analysis of single cell suspensions yielded from xenograft tumors. (F) Representative images of hematoxylin and eosin staining, and immunohistochemical staining of YTHDC2, Ki-67, cyclin D1, E-cadherin and $\mathrm{N}$-cadherin in xenograft tumors derived from nude mice. Scale bar, $50 \mu \mathrm{m}$; **P $<0.01$ vs. blank group. YTHDC2, YTH domain containing 2. 

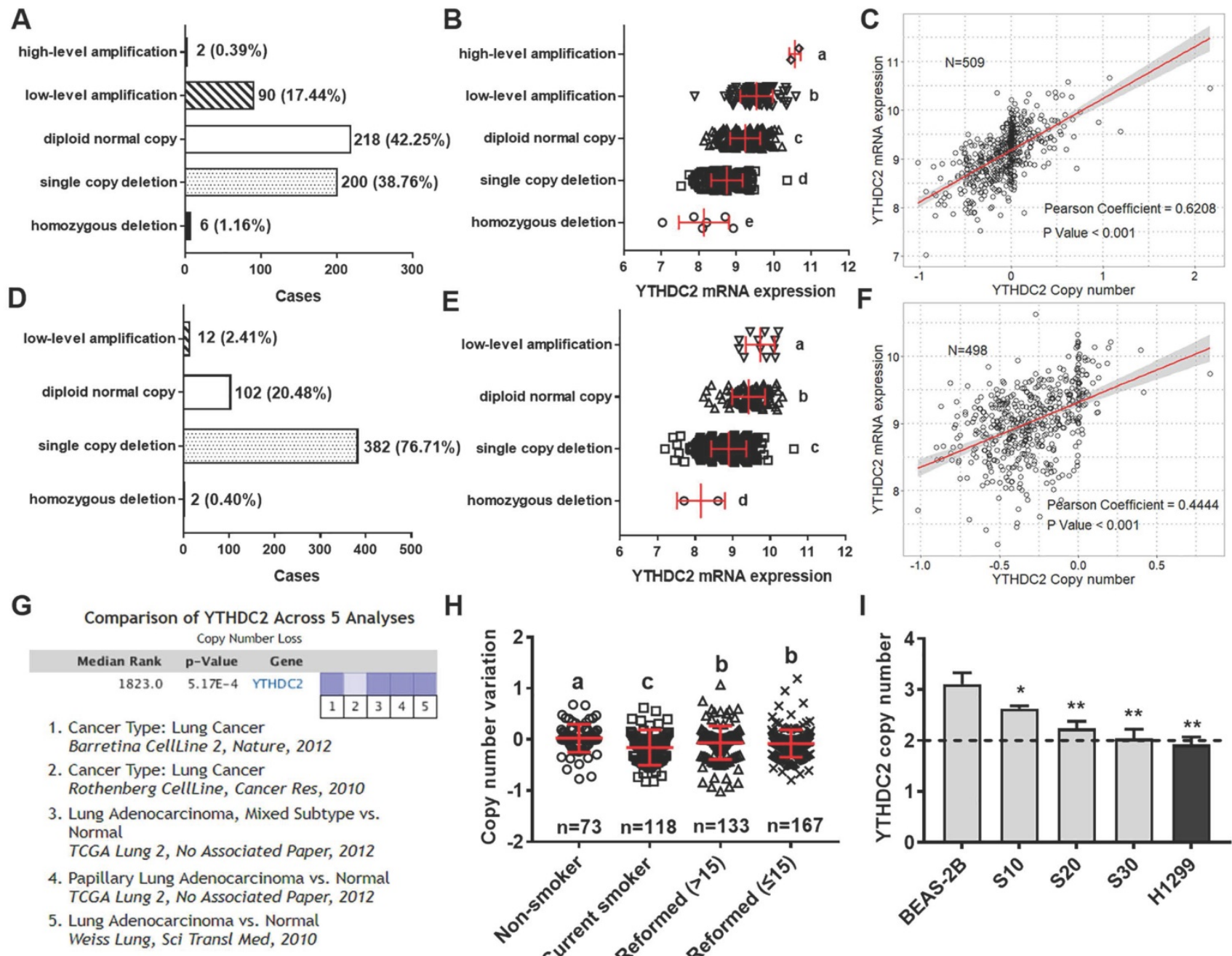

H
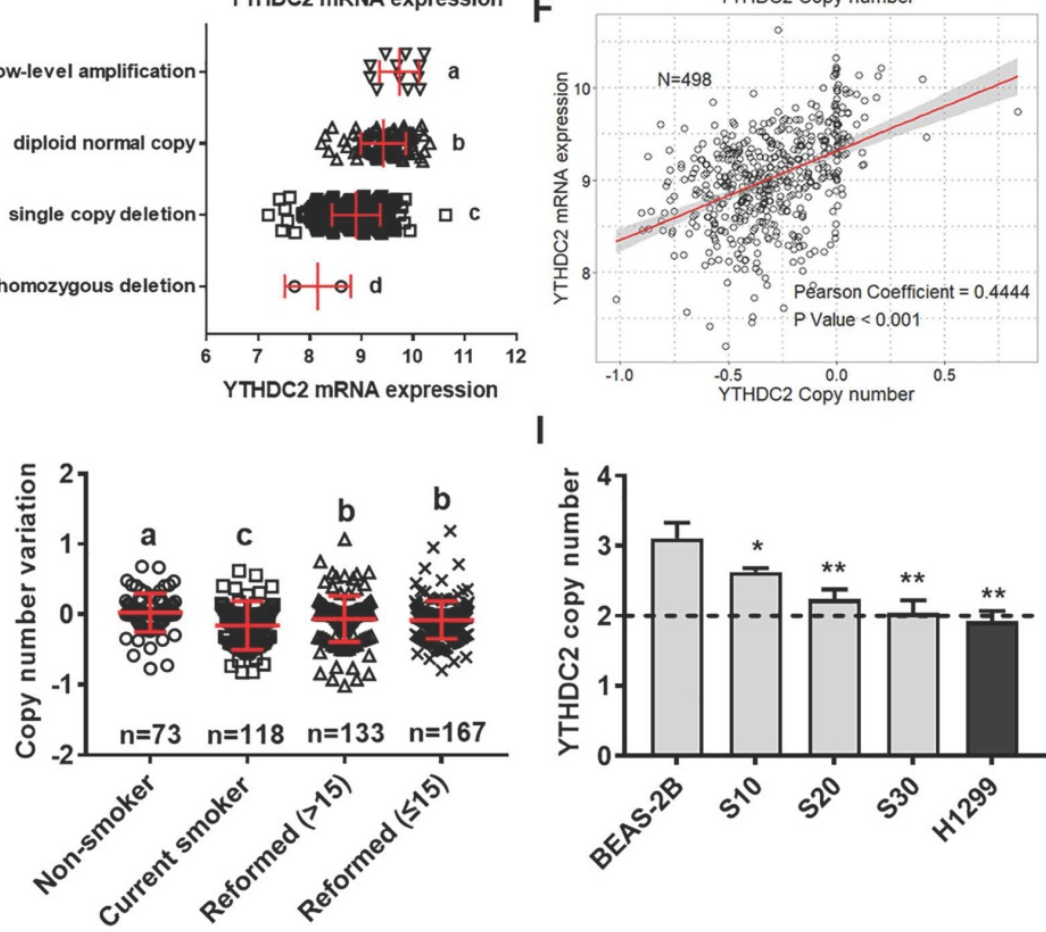

I

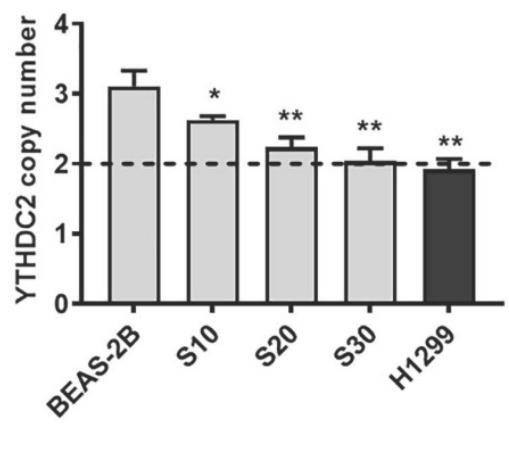

Figure 6. YTHDC2 mRNA expression was regulated by gene amplification. Distribution of patients with (A) LUAD and (D) LUSC with different YTHDC2 amplification status. YTHDC2 mRNA expression in (B) LUAD and (E) LUSC tissues with different YTHDC2 amplification status. Different letters (a, b, c and d) represent statistically significant group differences. Pearson's correlation analysis revealed a significant positive correlation between YTHDC2 mRNA expression and copy numbers in (C) LUAD and (F) LUSC. The line represents linear regression of data (LUAD: $y=1.065 x+9.177, R^{2}=0.385$; LUSC: $y=0.965 x+9.318, R^{2}=0.198$ ). (G) The Oncomine datasets for the corresponding YTHDC2 copy numbers in lung cancer were obtained with a threshold $\mathrm{P}=0.001$ and $\geq 2$ fold-change. The data in the graphic show significant downregulation (blue column) of $Y T H D C 2$ copy numbers in lung cancer versus normal tissue. The intensity of the blue color represents the respective levels of YTHDC2 copy number. (H) Copy number variation in LUAD samples with different smoking histories. Different letters (a, b, c and d) represent statistically significant group differences. (I) Copy number variation of YTHDC2 in BEAS-2B cells and cigarette smoke-exposed cells (grey block), as well as in two lung cancer cell lines (black block). The dotted line (copy number $=2)$ represents the copy number of the reference gene RNase P. *P<0.05, ** $\mathrm{P}<0.01$ vs. BEAS-2B cells. YTHDC2, YTH domain containing 2; LUSC, lung squamous cell carcinoma; LUAD, lung adenocarcinoma.

To verify the association between $Y T H D C 2$ copy number and smoking status, the present study analyzed the YTHDC2 genetic amplification in patients with lung cancer with different smoking histories. Compared with that of individuals who never smoked, the YTHDC2 copy number was significantly lower in current smokers and reformed smokers. Furthermore, the copy number was markedly increased in reformed smokers compared with that of current smokers, but it was still significantly lower than that of lifelong non-smokers (Figure 6H). The copy number variation of YTHDC2 was further verified in CS-exposed BEAS-2B cells and several lung cancer cell lines using TaqMan qPCR. The YTHDC2 copy numbers in CS-exposed cells (S10,
S20 and S30) and the NSCLC cell line (H1299) was lower than that in normal cells (Figure 6I). However, Kaplan-Meier survival and pathological analyses showed no significant prognostic effect in either LUAD or LUSC (Figure S8). Furthermore, no correlation was found between YTHDC2 copy numbers and pathological features (Table S3).

\section{CYLD is a downstream target of YTHDC2}

To further identify the downstream regulatory pathways of YTHDC2, RIP sequencing was performed to obtain the mRNAs that YTHDC2 can directly combine with. A total of 2,850 mRNAs were screened that were considered capable of binding to YTHDC2 under the criteria of $\mathrm{P}<0.05$ and $\log 2$ (fold-change) $>1$ (Figure 7A). Enrichment analysis 
showed that these mRNAs were significantly enriched in several important signaling pathways, including the 'Wnt signaling pathway', 'NF-kB-inducing kinase/NF-kB signaling', 'cell cycle' and 'cell-cell adhesion' (Figure 7B). Notably, in the intersection of YTHDC2 RIP-enriched mRNAs, YTHDC2-related mRNAs, upregulated DEPs and TSGs, only one gene named CYLD was obtained (Figure 7C). RIP PCR assay verified the combination of YTHDC2 and CYLD transcript (Figure S7C). According to correlation analysis, the CYLD mRNA expression level was significantly correlated with YTHDC2 in both LUAD and LUSC (Figure S7A and B). Consistent with YTHDC2, the CYLD mRNA expression in lung cancer tissues was lower than that in normal adjacent tissues in GSE32665 (Figure S9A) and GSE19188 (Figure S9B), as well as in GEPIA online tool (Figure S9C). Moreover, the Kaplan-Meier survival analysis revealed that lung cancer patients with high CYLD expression have the better prognosis (Figure S9D). When considering smoking history, CYLD mRNA expression was significantly lower in tumor tissues from smokers than from non-smokers (Figure S9E and F). In addition, CYLD was significantly downregulated in CS-exposed cells (S10, S20 and S30) at the mRNA and protein levels compared with the expression levels in unexposed BEAS-2B cells (Figure S9G-I).

RT-qPCR and western blot analyses showed a significant upregulation of CYLD expression in YTHDC2-overexpressing H1299 cells but not in YTHDC2-knockdown cells (Figure 7D and E). In addition, CYLD was identified as a negative regulator of NF-kB signaling [46]. The results of western blotting and immunofluorescence analyses suggested that NF-kB p65 was significantly accumulated in the nucleus, but was unchanged in total cells (Figure 7E and $\mathbf{G})$. In addition, the results of EMSA assay suggested decreased NF- $\mathrm{kB}$ activity in YTHDC2-overexpressing H1299 cells, while NF-кB activity was increased in YTHDC2-knockdown cells (Figure 7F). The RNA decay assay demonstrated that, compared with the findings in the blank control, YTHDC2 overexpression significantly slowed down the degradation of CYLD mRNA, whereas YTHDC2 knockdown significantly promoted its degradation (Figure 7H). In xenograft tumors, CYLD was upregulated, while NF-kB p65 was downregulated in the pYTHDC2 group compared with the findings in the blank group (Figure 7I).

\section{YTHDC2 regulates CYLD through $\mathbf{m}^{6} \mathrm{~A}$ modification}

According to the SRAMP online tool, 4 predicted $\mathrm{m}^{6} \mathrm{~A}$ methylated sites with markedly high confidence was found among the CYLD mRNA sequence (Figure 8A). To validate these $\mathrm{m}^{6} \mathrm{~A}$ sites, meRIP assay was used to obtain the methylated RNA, followed by qPCR and PCR. The qPCR and PCR results suggested that, after normalization of $\mathrm{m}^{6} \mathrm{~A}$ signals of specific segment to input, $4 \mathrm{~m}^{6} \mathrm{~A}$ sites (sites 2-5) from the 5 predicted regions with markedly high confidence showed more elevated levels, suggesting that the $\mathrm{m}^{6} \mathrm{~A}$ modification was present in the sequence of these sites (Figure 8B and $\mathrm{C}$ ). To further verify whether CYLD mRNA was regulated by $\mathrm{m}^{6} \mathrm{~A}$ modification, global $\mathrm{m}^{6} \mathrm{~A}$ methylation was modified by knocking down the $\mathrm{m}^{6} \mathrm{~A}$ methylases METTL3/14 and demethylases FTO/ALKBH5, and by treatment with the global methylation inhibitor 3-deazaadenosine (DAA) and the FTO inhibitor meclofenamic acid (MA). The results demonstrated that, when decreasing the $\mathrm{m}^{6} \mathrm{~A}$ level by knocking down METTL3/14 or by treatment with 3-DAA, the relative protein expression level of CYLD showed a significant decrease. Instead, when increasing the $\mathrm{m}^{6} \mathrm{~A}$ level by knocking down FTO/ALKBH5 or by treatment with MA, the CYLD relative protein expression level was markedly upregulated (Figure 8D-G). Importantly, the upregulated CYLD protein expression triggered by YTHDC2 overexpression could be significantly reduced by 3-DAA treatment, suggesting that YTHDC2 regulates CYLD stability through $\mathrm{m}^{6} \mathrm{~A}$ modification (Figure 8H and I).

\section{Discussion}

Members of the YTH domain family of proteins serve as crucial regulators of gene expression by specifically recognizing and binding to RNAs containing $\mathrm{m}^{6} \mathrm{~A}$ [47]. YTHDC1 has been shown to play an important regulatory role in pre-mRNA splicing in the nucleus, and YTHDF1, YTHDF2 and YTHDF3 have been found to play a synergistic role in promoting the effective translation and degradation of specific $\mathrm{m}^{6} \mathrm{~A}$-containing RNA in the cytoplasm [17, 18]. It is noteworthy that YTHDC2, the fifth member of the YTH protein family, exists both in the cytoplasm and in the nucleus. It has been demonstrated that YTHDC2 can modulate the expression and degradation of specific mRNAs in different types of cells [47]. YTHDC2 has been recognized to be associated with the development of certain types of cancer, including HCC [27] and colon cancer [26]. The present study used the GEPIA online tool to analyze the expression of YTHDC2, and found it to be significantly decreased in several solid tumors, including the two most common subtypes of lung cancer, compared with its expression in normal tissues. 


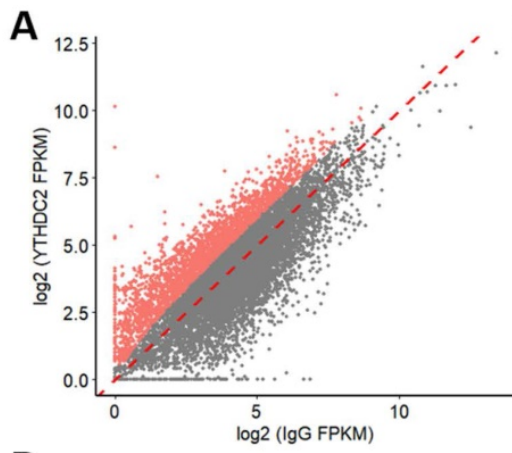

D

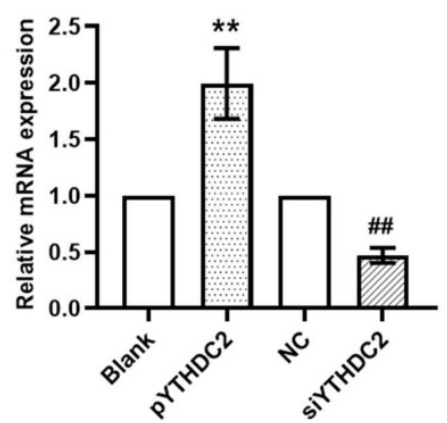

G
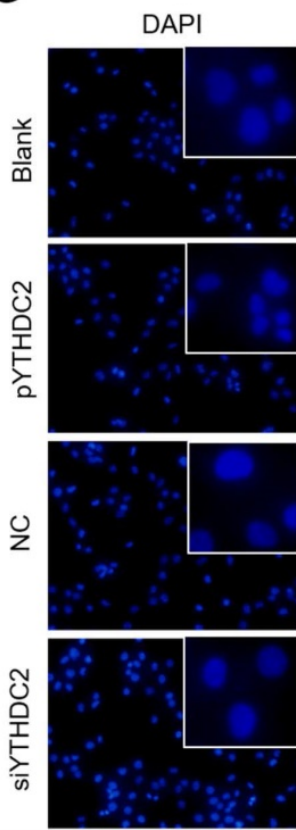

B

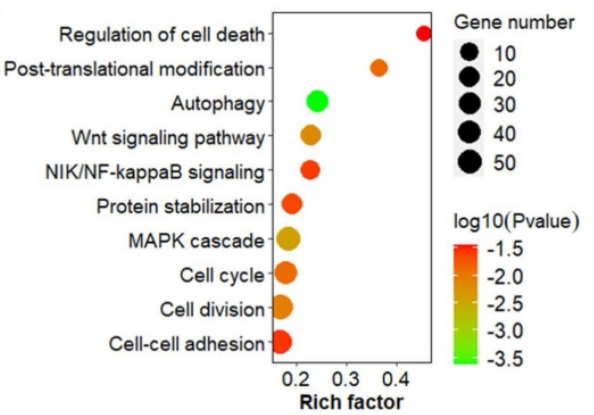

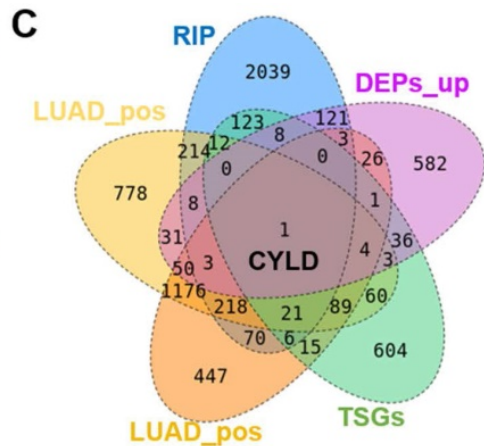

E

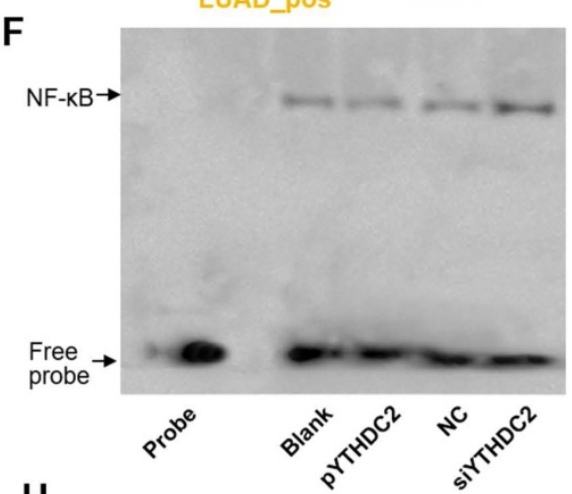

H

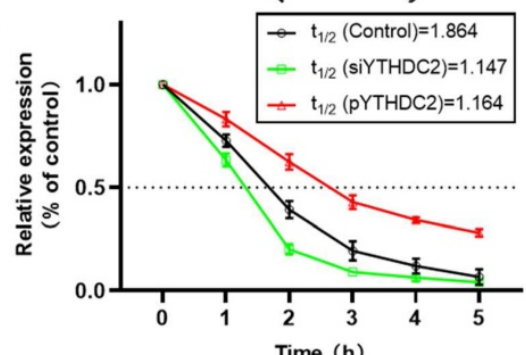

Blank

pYTHDC2

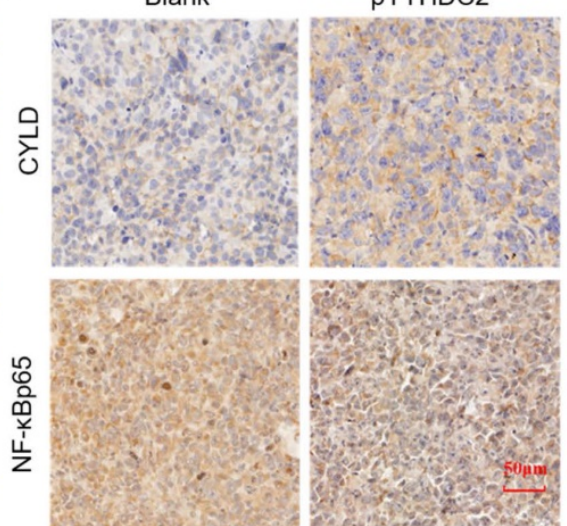

Figure 7. YTHDC2 promotes CYLD mRNA stability and inhibits NF-KB activity. (A) Scatter plot showing the mRNA transcripts identified by YTHDC2 RIP-sequencing, and transcripts that were significantly enriched are marked in red. (B) Kyoto Encyclopedia of Genes and Genomes pathway enrichment analysis of the mRNAs significantly enriched by YTHDC2 RIP. (C) Venn diagram showing the intersection of the YTHDC2 RIP-enriched mRNAs, YTHDC2-related mRNAs, upregulated differentially expressed proteins and tumor suppressor genes. (D) Relative mRNA expression level of CYLD in YTHDC2-overexpressing and knocked down H1299 cells. * $P<0.01$ vs. blank group, \#P<0.01 vs. negative control group. (E) Relative protein expression level of CYLD in YTHDC2-overexpressing and knocked down H1299 cells. (F) RNA decay assay for CYLD mRNA stability upon YTHDC2 overexpression and knockdown in H1299 cells. (G) Immunostaining analysis of NF-kB p65 and CYLD in YTHDC2-overexpressing and -knockdown H1299 cells. $(\mathrm{H})$ The DNA-binding activity of NF- $\mathrm{KB}$ in YTHDC2-overexpressing and -knockdown H1299 cells was measured by electrophoretic mobility shift assay using the biotin-labeled consensus NF- $\kappa B$-binding sequence. (I) Immunohistochemistry staining of NF- $\mathrm{BB}$ p65 and CYLD in xenograft tumors derived from nude mice. RIP, RNA immunoprecipitation; YTHDC2, YTH domain containing 2; CYLD, cylindromatosis.

The present study demonstrated that the expression of YTHDC2 was significantly downregulated in CS-exposed BEAS-2B cells. Based on bioinformatics and tissue array IHC analyses, the mRNA and protein expression of YTHDC2 was downregulated in lung cancer tissues compared with that of normal tissues. It was found that the expression of YTHDC2 was significantly lower in smoking patients than in non-smoking patients, which was consistent with the present in vitro 
experiments, suggesting that smoking could reduce the expression of YTHDC2. These results suggested that YTHDC2 is an important smoking-related gene in the regulation of the malignant potential of lung cancer cells. The current results showed different YTHDC2 expression profiles and prognostic significance in patients with lung cancer. However, the precise signaling pathways involved in the biological processes still need to be defined. YTHDC2 has been reported to contribute to colon cancer metastasis by promoting the translation of HIF-1a and its related pathways. The DEPs in YTHDC2-knockdown cells were linked to translation initiation, RNA splicing and RNA stability, which is consistent with the previously reported role of YTHDC2 in transcription [47]. The present results also showed that these DEPs are correlated with hypoxia, which occurs pathologically in solid tumors, and induces angiogenesis and tumor cell migration [48]. In addition, the bioinformatics and proteomics analyses demonstrated that YTHDC2 contributed to the initiation and progression of lung cancer. Decreased YTHDC2 expression in normal BEAS-2B lung cells and H1299 LUAD cells significantly enhanced cell migration. When YTHDC2 was knocked down in BEAS-2B cells, the expression of the epithelial marker CDH1 was significantly downregulated, while the expression of the mesenchymal marker $\mathrm{CDH} 2$ was significantly upregulated. Importantly, the xenograft animal model further validated the tumor-suppressor effect of YTHDC2 on lung cancer cell tumorigenesis in vivo. These observations suggested that decreased expression of YTHDC2 contributes to enhanced EMT ability, which is closely associated with the metastasis and progression of tumors.

A

Prediction score of $\mathrm{m}^{6} \mathrm{~A}$ distribution in the CYLD sequence
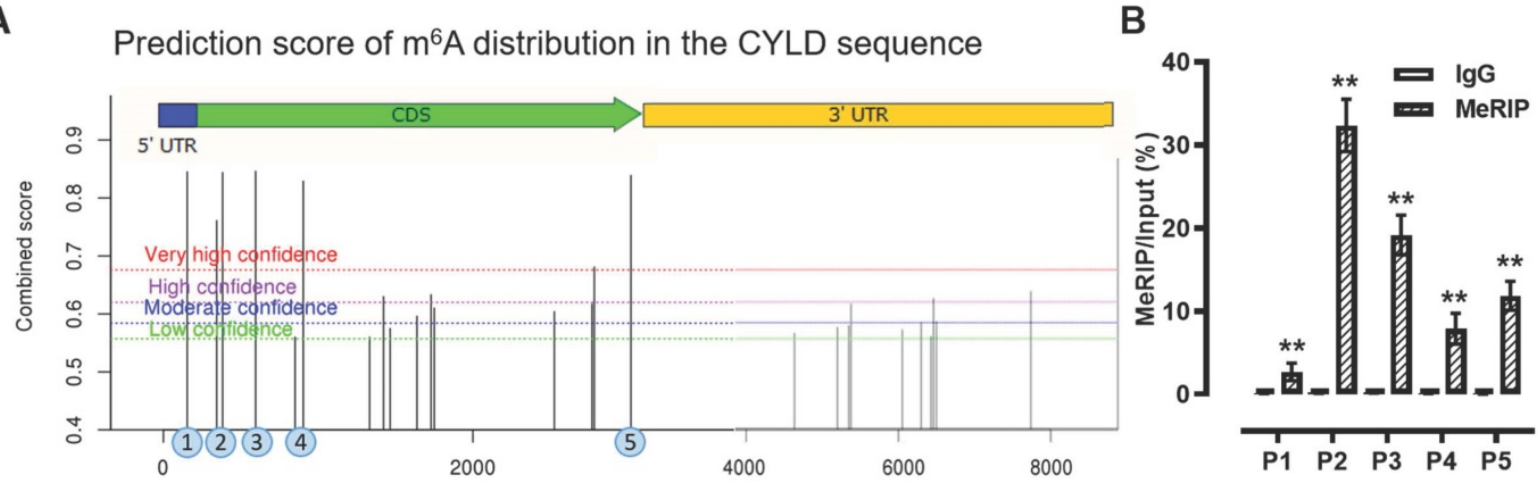

C

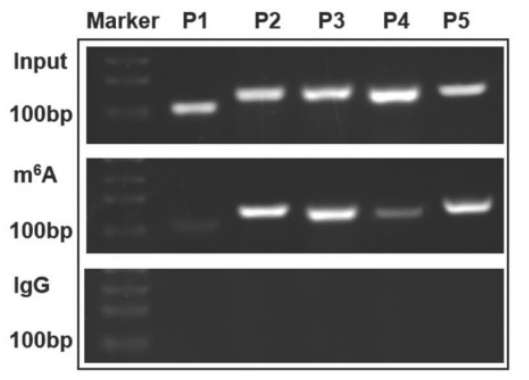

$\mathbf{F}$

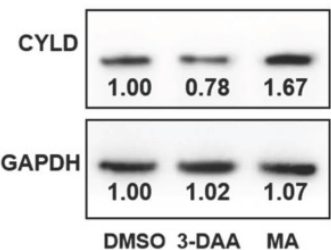

G

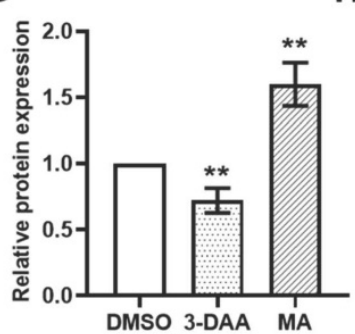

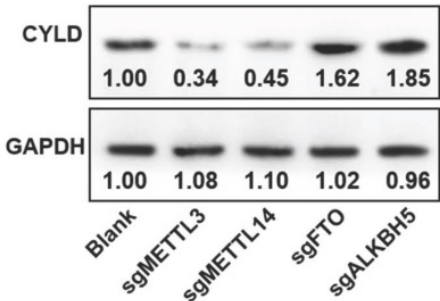

$\mathbf{H}$

E

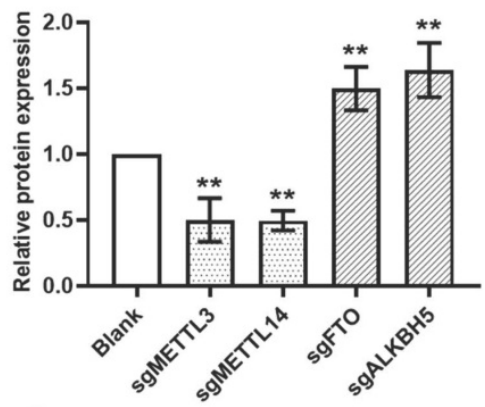

I

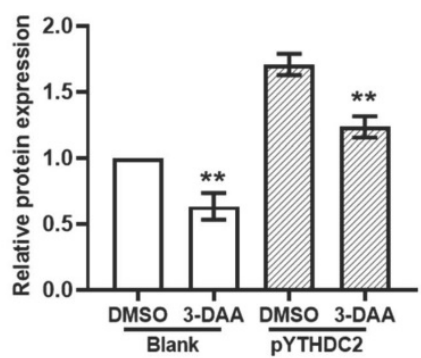

Figure 8. YTHDC2 regulates the stability of $C Y L D$ through $\mathrm{m}^{6} \mathrm{~A}$ modification. (A) Prediction score of $\mathrm{m}^{6} \mathrm{~A}$ distribution in $C Y L D$ sequence according to the sequence-based RNA adenosine methylation site predictor online tool. (B) meRIP-quantitative PCR and (C) meRIP-PCR results showed amplification of sites 2 to 5 , indicating m ${ }^{6} A$ modification in these four segments. Anti-lgG antibody was used as control. (D and E) Western blot results showing the relative protein expression level of CYLD in METTL3, METTLI4, FTO and ALKBH5 knocked down H1299 cells. (F and G) Western blot result showing the relative protein expression level of CYLD in H1299 cells treated with DAA, a global methylation inhibitor, and with meclofenamic acid, a FTO inhibitor. (H and I) Western blot results showing the relative protein expression level of CYLD in YTHDC2-overexpressing H1299 cells treated with or without 3-DAA. YTHDC2, YTH domain containing 2; CYLD, cylindromatosis; meRIP, m6 methylated RNA immunoprecipitation; m6 ${ }^{6}$, N6-methyladenosine; METTL, methyltransferase-like; FTO, fat mass and obesity-associated protein; DAA, 3-deazaadenosine. 
Variation in gene copy number of $>50 \mathrm{bp}$ in length is generally considered to play an essential role in the development of human tumors [49]. The loss of TSGs and the gain of proto-oncogenes were shown to contribute to cancer development $[50,51]$. It has been reported that the copy numbers and expression levels of programmed death-ligand 1 were both increased in smoking-related NSCLC [52]. Another study revealed that there was a significant correlation between the expression and copy number variation of EMT-related genes in numerous tumor samples of different cancer types [53]. In the present study, YTHDC2 expression was significantly correlated with copy number, and a decreased YTHDC2 copy number was highly associated with patient smoking history. Nevertheless, increased YTHDC2 copy number had no prognostic value in patients with lung cancer, since there was no significant correlation between YTHDC2 copy number and pathological features.

CYLD was reported as a tumor suppressor that participates in the occurrence and development of multiple cancer types, including pancreatic cancer [54], colon carcinoma [55], breast cancer [56] and HCC [57]. CYLD is a deubiquitinating enzyme that inhibits polyubiquitin-dependent pathways, including the NF-kB signaling pathway, which can promote cell growth and inflammatory [58]. However, as an important TSG, the upstream regulatory mechanism of CYLD in cancer remains unclear. A previous study reported that CYLD can be regulated by lncRNA CRAL/miR-505 in human gastric cancer cells to reverse cisplatin resistance [59]. Several other miRNAs were also reported to regulate CYLD in multiple cancer types. For example, in colon cancer, miR-181b was able to target CYLD to inhibit the NF- $\kappa \mathrm{B}$ signaling pathway [60], while, in cervical cancer, miR-501 can target CYLD to regulate cell proliferation, migration and invasion [61]. The present study identified multiple $\mathrm{m}^{6} \mathrm{~A}$ methylation sites in CYLD transcripts from an epigenetics perspective, which were recognized and bound by the $\mathrm{m}^{6} \mathrm{~A}$ reader YTHDC2, thus further contributing to CYLD mRNA stability. YTH domain-containing readers have been reported to be able to regulate the splicing, translation or stability of specific $\mathrm{m}^{6} \mathrm{~A}$ modified mRNAs [47]. A previous study demonstrated that YTHDC2 can suppress LUAD tumorigenesis via promoting solute carrier family 7 member 11 (SLC7A11) decay [62]. The current study identified CYLD as a target of YTHDC2 based on bioinformatics analysis, RIP sequencing and proteomic analysis, and found that YTHDC2 is able to promote CYLD mRNA stability.

In conclusion, the present results indicated that the downregulation of smoking-related YTHDC2 was associated with enhanced proliferation and migration of lung cancer cells, and appeared to be regulated by DNA copy number variation. CYLD/NF-kB is a vital downstream pathway that contributes to the tumorsuppressor role of YTHDC2 in lung cancer cells. Further in vitro and in vivo studies are needed to gain insights into additional $\mathrm{m}^{6} \mathrm{~A}$-dependent downstream mechanisms of YTHDC2 in lung cancer.

\section{Abbreviations}

SCLC: small cell lung cancer; NSCLC: non-small cell lung cancer; LUSC: lung squamous cell carcinoma; LUAD: lung adenocarcinoma; OS: overall survival; HCC: hepatocellular carcinoma; GEO: Gene Expression Omnibus; TCGA: The Cancer Gene Atlas; UCSC: University of California Santa Cruz; RSEM: RNA-seq expression value; HRs: hazard ratios; CIs: confidence intervals; TSGs: tumor suppressor genes; MRGs: metastasis-related genes; cDNA: complementary DNA; ATCC: American Type Culture Collection; FBS: fetal bovine serum; CDS: coding sequence; SD: standard deviation; HPA: Human Protein Atlas; EMT: epithelial-mesenchymal transformation; CDH1: E-cadherin; $\mathrm{CDH} 2$ : $\mathrm{N}$-Cadherin; CNV: copy number variation; RIP: RNA immunoprecipitation; meRIP: $\mathrm{m}^{6} \mathrm{~A}$ methylated RNA immunoprecipitation; EMSA: Electrophoretic mobility shift assay.

\section{Supplementary Material}

Supplementary figures and tables. http://www.ijbs.com/v17p2633s1.pdf

\section{Acknowledgements}

\section{Funding}

This study was supported by the National Natural Science Foundation of China (81573178 and 81770085). The study was also supported by Jiangsu Key Laboratory of Preventive and Translational Medicine for Geriatric Diseases as well as the Priority Academic Program Development of Jiangsu Higher Education Institutions (PAPD).

\section{Data Statement}

The TCGA LUAD and LUSC datasets analyzed for this study can be obtained from UCSC Xena (https://xenabrowser.net/datapages/). All GEO datasets analyzed for this study can be obtained from Gene Expression Omnibus (GEO, http://www.ncbi. nlm.nih.gov/geo/).

\section{Author Contributions}

JL and RC conceived and designed the study. JW, LT and BJ performed and analyzed the experiments. JW, XY, RY, NO, XY and XC assisted to 
collect and analyze the data. JW and LT wrote the manuscript. JT was of immense help in the modification of the manuscript. All authors read and approved the final manuscript.

\section{Competing Interests}

The authors have declared that no competing interest exists.

\section{References}

1. Siegel RL, Miller KD, Jemal A. Cancer statistics, 2018. CA Cancer J Clin. 2018; 68: 7-30.

2. Chang JT, Lee YM, Huang RS. The impact of the Cancer Genome Atlas on lung cancer. Transl Res. 2015; 166: 568-85.

3. Boolell V, Alamgeer M, Watkins DN, Ganju V. The Evolution of Therapies in Non-Small Cell Lung Cancer. Cancers (Basel). 2015; 7: 1815-46.

4. Doll R, Peto R, Boreham J, Sutherland I. Mortality in relation to smoking: 50 years' observations on male British doctors. BMJ. 2004; 328: 1519.

5. Warren GW, Cummings KM. Tobacco and lung cancer: risks, trends, and outcomes in patients with cancer. Am Soc Clin Oncol Educ Book. 2013: 359-64.

6. Gallant JN, Lovly CM. Established, emerging and elusive molecular targets in the treatment of lung cancer. J Pathol. 2018; 244: 565-77.

7. Wu DJ, Wang XD. Application of clinical bioinformatics in lung cancer-specific biomarkers. Cancer Metast Rev. 2015; 34: 209-16.

8. Beane J, Vick J, Schembri F, Anderlind C, Gower A, Campbell J, et al. Characterizing the Impact of Smoking and Lung Cancer on the Airway Transcriptome Using RNA-Seq. Cancer Prev Res. 2011; 4: 803-17.

9. Malhotra J, Malvezzi M, Negri E, La Vecchia C, Boffetta P. Risk factors for lung cancer worldwide. Eur Respir J. 2016; 48: 889-902

10. Du H, Sun J, Chen Z, Nie J, Tong J, Li J. Cigarette smoke-induced failure of apoptosis resulting in enhanced neoplastic transformation in human bronchial epithelial cells. J Toxicol Environ Health, Part A. 2012; 75: 707-20.

11. Huang H, Ji Y, Zhang J, Su Z, Liu M, Tong J, et al. Aberrant DNA methylation in radon and/or cigarette smoke-induced malignant transformation in BEAS-2B human lung cell line. J Toxicol Environ Health, Part A. 2017; 80: 1321-30.

12. Liu J, Eckert MA, Harada BT, Liu SM, Lu Z, Yu K, et al. m(6)A mRNA methylation regulates AKT activity to promote the proliferation and tumorigenicity of endometrial cancer. Nat Cell Biol. 2018; 20: 1074-83.

13. Ma JZ, Yang F, Zhou CC, Liu F, Yuan JH, Wang F, et al. METTL14 suppresses the metastatic potential of hepatocellular carcinoma by modulating $\mathrm{N}(6)$ -methyladenosine-dependent primary MicroRNA processing. Hepatology. 2017; 65: 529-43.

14. Choe J, Lin S, Zhang W, Liu Q, Wang L, Ramirez-Moya J, et al. mRNA circularization by METTL3-eIF3h enhances translation and promotes oncogenesis. Nature. 2018; 561: 556-60.

15. Wang $\mathrm{X}, \mathrm{He} \mathrm{C}$. Reading RNA methylation codes through methyl-specific binding proteins. RNA Biol. 2014; 11: 669-72.

16. Zhang ZY, Theler D, Kaminska KH, Hiller M, de la Grange P, Pudimat R, et al. The YTH Domain Is a Novel RNA Binding Domain. J Biol Chem. 2010; 285: 14701-10.

17. Wang X, Zhao BS, Roundtree IA, Lu Z, Han D, Ma H, et al. N(6)-methyladenosine Modulates Messenger RNA Translation Efficiency. Cell. 2015; 161: 1388-99.

18. Shi H, Wang X, Lu Z, Zhao BS, Ma H, Hsu PJ, et al. YTHDF3 facilitates translation and decay of N(6)-methyladenosine-modified RNA. Cell Res. 2017; 27: $315-28$

19. Tuck MT, James CB, Kelder B, Kopchick JJ. Elevation of internal 6-methyladenine mRNA methyltransferase activity after cellular transformation. Cancer Lett. 1996; 103: 107-13.

20. Bai Y, Yang C, Wu R, Huang L, Song S, Li W, et al. YTHDF1 Regulates Tumorigenicity and Cancer Stem Cell-Like Activity in Human Colorectal Carcinoma. Front Oncol. 2019; 9: 332.

21. Yang Z, Li J, Feng G, Gao S, Wang Y, Zhang S, et al. MicroRNA-145 Modulates N(6)-Methyladenosine Levels by Targeting the 3'-Untranslated mRNA Region of the N(6)-Methyladenosine Binding YTH Domain Family 2 Protein. J Biol Chem. 2017; 292: 3614-23.

22. Li J, Meng $\mathrm{S}, \mathrm{Xu} \mathrm{M}$, Wang $\mathrm{S}, \mathrm{He} \mathrm{L}, \mathrm{Xu} \mathrm{X}$, et al. Downregulation of $\mathrm{N}(6)$-methyladenosine binding YTHDF2 protein mediated by miR-493-3p suppresses prostate cancer by elevating N(6)-methyladenosine levels. Oncotarget. 2018; 9: 3752-64.

23. Hsu PJ, Zhu YF, Ma HH, Guo YH, Shi XD, Liu YY, et al. Ythdc2 is an N-6-methyladenosine binding protein that regulates mammalian spermatogenesis. Cell Res. 2017; 27: 1115-27.

24. Jain D, Puno MR, Meydan C, Lailler N, Mason CE, Lima CD, et al. ketu mutant mice uncover an essential meiotic function for the ancient RNA helicase YTHDC2. Elife. 2018; 7 .

25. Abby E, Tourpin S, Ribeiro J, Daniel K, Messiaen S, Moison D, et al Implementation of meiosis prophase I programme requires a conserved retinoid-independent stabilizer of meiotic transcripts. Nat Commun. 2016; 7: 10324

26. Tanabe A, Tanikawa K, Tsunetomi M, Takai K, Ikeda H, Konno J, et al. RNA helicase YTHDC2 promotes cancer metastasis via the enhancement of the efficiency by which HIF-1alpha mRNA is translated. Cancer Lett. 2016; 376: $34-42$.

27. Tanabe A, Konno J, Tanikawa K, Sahara H. Transcriptional machinery of TNF-alpha-inducible YTH domain containing 2 (YTHDC2) gene. Gene. 2014; 535: 24-32.

28. Tang Z, Li C, Kang B, Gao G, Li C, Zhang Z. GEPIA: a web server for cancer and normal gene expression profiling and interactive analyses. Nucleic Acids Res. 2017; 45: W98-W102

29. Kim IJ, Quigley D, To MD, Pham P, Lin K, Jo B, et al. Rewiring of human lung cell lineage and mitotic networks in lung adenocarcinomas. Nat Commun. 2013; 4: 1701.

30. Hou J, Aerts J, den Hamer B, van Ijcken W, den Bakker M, Riegman P, et al. Gene expression-based classification of non-small cell lung carcinomas and survival prediction. PLoS ONE. 2010; 5: e10312.

31. Sato M, Larsen JE, Lee W, Sun H, Shames DS, Dalvi MP, et al. Human Lung Epithelial Cells Progressed to Malignancy through Specific Oncogenic Manipulations. Mol Cancer Res. 2013; 11: 638-50.

32. Rousseaux S, Debernardi A, Jacquiau B, Vitte AL, Vesin A, Nagy-Mignotte H, et al. Ectopic activation of germline and placental genes identifies aggressive metastasis-prone lung cancers. Sci Transl Med. 2013; 5: 186ra66.

33. Varghese F, Bukhari AB, Malhotra R, De A. IHC Profiler: An Open Source Plugin for the Quantitative Evaluation and Automated Scoring of Immunohistochemistry Images of Human Tissue Samples. PLoS ONE. 2014; 9: e96801.

34. Gyorffy B, Surowiak P, Budczies J, Lanczky A. Online Survival Analysis Software to Assess the Prognostic Value of Biomarkers Using Transcriptomic Data in Non-Small-Cell Lung Cancer. PLoS ONE. 2013; 8.

35. Vasaikar S, Straub P, Wang J, Zhang B. LinkedOmics: Analyzing multi-omics data within and across 32 cancer types. Cancer Res. 2018; 78.

36. Huang DW, Sherman BT, Lempicki RA. Systematic and integrative analysis of large gene lists using DAVID bioinformatics resources. Nat Protoc. 2009; 4: 44-57.

37. Zhao M, Sun JC, Zhao ZM. TSGene: a web resource for tumor suppressor genes. Nucleic Acids Res. 2013; 41: D970-D6.

38. Zheng G, Ma Y, Zou Y, Yin A, Li W, Dong D. HCMDB: the human cancer metastasis database. Nucleic Acids Res. 2018; 46: D950-D5.

39. Shannon P, Markiel A, Ozier O, Baliga NS, Wang JT, Ramage D, et al. Cytoscape: a software environment for integrated models of biomolecular interaction networks. Genome Res. 2003; 13: 2498-504.

40. Livak KJ, Schmittgen TD. Analysis of relative gene expression data using real-time quantitative PCR and the 2(T)(-Delta Delta C) method. Methods. 2001; 25: 402-8.

41. Mermel CH, Schumacher SE, Hill B, Meyerson ML, Beroukhim R, Getz G. GISTIC2.0 facilitates sensitive and confident localization of the targets of focal somatic copy-number alteration in human cancers. Genome Biol. 2011; 12: R41.

42. Rhodes DR, Yu J, Shanker K, Deshpande N, Varambally R, Ghosh D, et al. ONCOMINE: a cancer microarray database and integrated data-mining platform. Neoplasia. 2004; 6: 1-6.

43. Zhao J, Sun BK, Erwin JA, Song JJ, Lee JT. Polycomb proteins targeted by a short repeat RNA to the mouse X chromosome. Science. 2008; 322: 750-6.

44. Ratnadiwakara M, Änkö M-L. mRNA Stability Assay Using Transcription Inhibition by Actinomycin D in Mouse Pluripotent Stem Cells. Bio-protocol. 2018; 8: e3072.

45. Zhou Y, Zeng P, Li YH, Zhang Z, Cui Q. SRAMP: prediction of mammalian N6-methyladenosine (m6A) sites based on sequence-derived features. Nucleic Acids Res. 2016; 44: e91.

46. Brummelkamp TR, Nijman SM, Dirac AM, Bernards R. Loss of the cylindromatosis tumour suppressor inhibits apoptosis by activating NF-kappaB. Nature. 2003; 424: 797-801.

47. Kretschmer J, Rao H, Hackert P, Sloan KE, Hobartner C, Bohnsack MT. The $\mathrm{m}(6)$ A reader protein YTHDC2 interacts with the small ribosomal subunit and the 5'-3' exoribonuclease XRN1. RNA. 2018; 24: 1339-50.

48. Karakashev SV, Reginato MJ. Progress toward overcoming hypoxia-induced resistance to solid tumor therapy. Cancer Manag Res. 2015; 7: 253-64.

49. Conrad DF, Pinto D, Redon R, Feuk L, Gokcumen O, Zhang Y, et al. Origins and functional impact of copy number variation in the human genome. Nature. 2010; 464: 704-12.

50. Tran TN, Selinger CI, Kohonen-Corish MRI, McCaughan BC, Kennedy CW, O'Toole SA, et al. Fibroblast growth factor receptor 1 (FGFR1) copy number is an independent prognostic factor in non-small cell lung cancer. Lung Cancer. 2013; 81: 462-7.

51. Qiu ZW, Bi JH, Gazdar AF, Song K. Genome-wide copy number variation pattern analysis and a classification signature for non-small cell lung cancer. Gene Chromosome Canc. 2017; 56: 559-69.

52. Inoue $\mathrm{Y}$, Yoshimura $\mathrm{K}$, Mori $\mathrm{K}$, Kurabe $\mathrm{N}$, Kahyo $\mathrm{T}$, Mori $\mathrm{H}$, et al. Clinical significance of PD-L1 and PD-L2 copy number gains in non-small-cell lung cancer. Oncotarget. 2016; 7: 32113-28.

53. Zhao M, Liu Y, Qu H. Expression of epithelial-mesenchymal transition-related genes increases with copy number in multiple cancer types. Oncotarget. 2016; 7: 24688-99. 
54. Xie S, Wu Y, Hao H, Li J, Guo S, Xie W, et al. CYLD deficiency promotes pancreatic cancer development by causing mitotic defects. J Cell Physiol. 2019; 234: 9723-32.

55. Hellerbrand C, Bumes E, Bataille F, Dietmaier W, Massoumi R, Bosserhoff AK. Reduced expression of CYLD in human colon and hepatocellular carcinomas. Carcinogenesis. 2007; 28: 21-7.

56. Hayashi M, Jono H, Shinriki S, Nakamura T, Guo JY, Sueta A, et al. Clinical significance of CYLD downregulation in breast cancer. Breast Cancer Res Treat. 2014; 143: 447-57.

57. Pannem RR, Dorn C, Ahlqvist K, Bosserhoff AK, Hellerbrand C, Massoumi R. CYLD controls c-MYC expression through the JNK-dependent signaling pathway in hepatocellular carcinoma. Carcinogenesis. 2014; 35: 461-8.

58. Trompouki E, Hatzivassiliou E, Tsichritzis T, Farmer H, Ashworth A, Mosialos G. CYLD is a deubiquitinating enzyme that negatively regulates NF-kappa B activation by TNFR family members. Nature. 2003; 424: 793-6.

59. Wang ZD, Wang Q, Xu GF, Meng N, Huang XL, Jiang ZR, et al. The long noncoding RNA CRAL reverses cisplatin resistance via the miR-505/ CYLD/AKT axis in human gastric cancer cells. RNA Biol. 2020; 17: 1576-89.

60. Yang X, Sun Y, Zhang Y, Han S. Downregulation of miR181b inhibits human colon cancer cell proliferation by targeting CYLD and inhibiting the NFkappaB signaling pathway. Int J Mol Med. 2020; 46: 1755-64.

61. Sanches JGP, Xu YC, Yabasin IB, Li M, Lu Y, Xiu XX, et al. miR-501 is upregulated in cervical cancer and promotes cell proliferation, migration and invasion by targeting CYLD. Chem-Biol Interact. 2018; 285: 85-95.

62. Ma L, Chen T, Zhang X, Miao Y, Tian X, Yu K, et al. The m(6)A reader YTHDC2 inhibits lung adenocarcinoma tumorigenesis by suppressing SLC7A11-dependent antioxidant function. Redox Biol. 2021; 38: 101801. 